ZU-TH

December 2002

\title{
Consistent treatment of spin-1 mesons in the light-front quark model
}

\author{
Wolfgang Jaus \\ Institut für Theoretische Physik der Universität Zürich, Winterthurerstr. 190, CH-8057 Zürich \\ Switzerland
}

\begin{abstract}
We analyze the matrix element of the electroweak current between $q \bar{q}$ vector meson states in the framework of a covariant extension of the light-front formalism. The light-front matrix element of a one-body current is naturally associated with zero modes, which affect some of the form factors that are necessary to represent the Lorentz structure of the light-front integral. The angular condition contains information on zero modes, i.e., only if the effect of zero modes is accounted for correctly, is it satisfied. With plausible assumptions we derive from the angular condition several consistency conditions which can be used quite generally to determine the zero mode contribution of form factors. The correctness of this method is tested by the phenomenological success of the derived form factors. We compare the predictions of our formalism with those of the standard light-front approach and with available data. As examples we discuss the magnetic moment of the $\rho$, the coupling constant $g_{D^{*} D \pi}$, and the coupling constants of the pseudoscalar density, $g_{\pi}$ and $g_{K}$, which provide a phenomenological link between constituent and current quark masses.
\end{abstract}

PACS number(s): 12.39Ki, 13.25Ft, 13.40-f, 14.65Bt 


\section{Introduction}

The light-front formalism [1] has been used extensively in particle and nuclear physics. It provides a conceptually simple, phenomenological framework for the determination of the form factors of a relativistic composite system. For practical applications matrix elements of the current operator, which is approximated by a one-body current, are taken between states with the same number of constituents; these are, e.g., the confined constituent quark and antiquark meson states, the three-quark baryon states or the bound two-nucleon deuteron state. In general this approach does not permit an unambiguous determination of the physical form factors, for it is well known $[2,3,4]$ that the light-front calculation of the matrix element of a one-body current generates a 4-vector structure that is in general not covariant, since it contains a spurious dependence on the orientation of the lightfront. The light-front is defined in terms of the lightlike 4 -vector $\omega$ by the invariant equation $\omega \cdot x=0$. The special case $\omega=\left(\omega^{0}, \omega^{1}, \omega^{2}, \omega^{3}\right)=(1,0,0,-1)$ corresponds to the light-front or null-plane $\omega \cdot x=x^{+}=x^{0}+x^{3}=0$.

The 4-momenta of the initial and final states are therefore not enough to construct the Lorentz decomposition of the approximate matrix element, the unphysical 4 -vector $\omega$ is required too. This construction does not only lead to additional, unphysical form factors, but generates also a spurious dependence on $\omega$ of some of the form factors. This problem is closely associated with the violation of rotational invariance in the computation of the matrix element of a one-body current $[5,6]$.

However, full Lorentz covariance could be restored even at this level of approximation if it would be possible to include the effect of the associated zero modes [7]. In Ref. [8] we have investigated the relationship between a simple (but unrealistic) covariant model for $q \bar{q}$ mesons and its light-front representation in 1-loop order. In a frame where the momentum transfer $q^{+}=q^{0}+q^{3}=0$, the model permits the calculation of the $\delta(x)$ contributions, where $p_{1}^{\prime+}=x P^{\prime+}$ is the plus component of the constituent quark momentum and $P^{\prime+}$ is the plus component the momentum of the initial meson state. The $\delta(x)$ or zero mode terms are naturally associated with the light-front matrix element of a one-body current and restore full covariance. They minimally account for neglected interaction effects which is obvious from the work in Refs. $[9,10,11]$ where the zero mode contributions proportional to $\delta(x)$ have been interpreted as residues of virtual pair creation processes in the limit $q^{+}=0$. While these model calculations $[8,9,10,11]$ have been used to investigate the role of zero modes in a light-front approach, they did not provide a practical method for a realistic phenomenology. It is the purpose of the present work to develop a method that permits the calculation of the contribution of the zero modes associated with the matrix element of a one-body current and, consequently, a consistent determination of the physical form factors.

Note that the standard light-front (SLF) formalism uses only the matrix elements of the plus component of the one-body current and requires all constituents to be on their respective mass shells. These rules ignore the effect of zero modes and the 
covariance of the matrix element is lost. Nevertheless, the zero mode contributions can be avoided for transitions that involve only pseudoscalar mesons if the hadronic form factors are calculated from the plus components of the respective currents, but this is no longer true for composite spin-1 systems. An impressive example is provided by three different light-front calculations of the elastic electromagnetic form factors of the deuteron in Refs. [12, 13, 14]. The starting point in each case is the plus component of the matrix element of the one-body electromagnetic current, nevertheless the final results are different. Such an outcome is possible since the number of independent helicity amplitudes is larger than the number of physical form factors. However, the requirement of covariance imposes a nontrivial dynamic constraint on the helicity amplitudes which is known as the angular condition [12, 13]. If the angular condition is fulfilled, different prescriptions for the calculation of the form factors lead to the same result. The standard light-front approach in general violates the angular condition and thus signals again that it is not covariant.

An interesting analysis of the spin-1 system has recently been published by Bakker, Choi and Ji $[15,16]$. In Ref. [15] the frame dependence of the angular condition is investigated. In Ref. [16] spin-1 form factors are analyzed with respect to different prescriptions, different choices of polarization vectors and reference frames. In [16] a covariant model is used, which (as far as the triangle diagram is concerned) is similar to ours [8], to guide the corresponding light-front calculation. A particular prescription is found to derive the physical form factors, that is not affected by zero mode contributions and coincides exactly with the result of the covariant model calculation.

The derivation [16] of this outcome is model dependent and we shall show in the present paper that it is of general validity only for the simple $q \bar{q}$-vector meson vertex chosen in Ref. [16], but if the electromagnetic form factors of a vector meson, $F_{1}\left(q^{2}\right)$, $F_{2}\left(q^{2}\right)$ and $F_{3}\left(q^{2}\right)$, are calculated with the standard light-front $q \bar{q}$-vector meson vertex, the magnetic dipole form factor $F_{2}\left(q^{2}\right)$ requires zero mode contributions. Our analysis is based on the formalism which we have developed in Ref. [8]. At first we shall investigate electroweak transitions between $q \bar{q}$ mesons of spin 1 . The angular condition is shown to lead to several constraints on those parts of the matrix element of the one-body current that depend on $\omega$, and which must be completed by an appropriate account of zero modes. In this manner spurious contributions to form factors can be eliminated completely. The angular condition is of crucial importance for our analysis: it not only checks the covariance of a procedure, but it contains information on the effect of zero modes of which we shall take full advantage. This method permits a consistent light-front calculation of the transition form factors of vector mesons in the 1-loop approximation.

There is an alternative and quite different scheme to deal with the problem of the violation of covariance of the SLF formalism, which has been reviewed in Ref. [2] (we shall refer to it by the label CDKM). In order to treat the complete Lorentz structure of a hadronic matrix element the authors of Ref. [2] have developed a method to identify and separate spurious contributions and to determine the physical, i.e. $\omega$ - 
independent contributions to the hadronic form factors and coupling constants. We shall compare the results of our work with those of the CDKM approach.

In [8] we have found that in the determination of form factors one has to deal with two classes. There is one class of form factors like the charge form factor of a pseudoscalar meson, $V\left(q^{2}\right)$ and $A_{2}\left(q^{2}\right)$ for transitions between vector and pseudoscalar mesons, and the pseudoscalar coupling constant $f_{P}$ that are free of zero mode contributions. These form factors are predicted unambiguously in 1-loop order by the standard light-front approach, and every covariant extension of the SLF formalism (like the approach of this work or that of CDKM) must reproduce these results. Another class of form factors like $F_{2}\left(q^{2}\right), A_{1}\left(q^{2}\right)$ and the vector coupling constant $f_{V}$ are associated with zero modes. For their reliable prediction in 1-loop order the corresponding zero mode contribution must be known. It is somewhat surprising that the angular condition, which has been established for transitions between vector mesons, can be used not only to determine the zero mode contributions to the magnetic form factor of a vector meson $F_{2}\left(q^{2}\right)$, but also those to $f_{V}$ and $A_{1}\left(q^{2}\right)$. The form factors that we shall determine in such a manner are different from those obtained in the SLF or CDKM schemes, both of which ignore zero mode contributions.

In Sec.II we present a brief summary of the basic formalism for the treatment of the transition form factors for spin-1 mesons. We discuss the angular condition, and with plausible assumptions we derive from it several consistency conditions which can be used quite generally to determine effective zero mode contributions. The formal machinery required for the Lorentz decomposition of the light-front matrix element has been collected in the Appendices A and B. In Sec.III we use this method to calculate the zero mode contributions associated with $f_{V}$ and $A_{1}\left(q^{2}\right)$, and derive the formulas for these quantities in our formalism, and also in the SLF and CDKM schemes. In Sec.IV we take the magnetic moment of the rho meson and the coupling constants $g_{V P \pi}$ as examples to compare quantitative predictions of our approach with those of the SLF and CDKM schemes. We also briefly discuss the coupling constant of the pseudoscalar density, $g_{P}$, whose zero mode contribution can be determined by our method, and which provides a phenomenological link between constituent and current quark masses. We conclude this work in Sec.V with a summary of our analysis.

\section{Transition form factors for spin-1 mesons}

\subsection{Angular condition for electroweak transitions}

We shall generalize the procedure of [16] for the matrix element of an electroweak current $V^{\mu}=\bar{q}^{\prime \prime} \gamma^{\mu} q^{\prime}$ between initial and final $q \bar{q}$ vector meson states of 4-momentum, mass and helicity $\left(P^{\prime}, M^{\prime}, h^{\prime}\right)$ and $\left(P^{\prime \prime}, M^{\prime \prime}, h^{\prime \prime}\right)$, respectively. Its most general form is represented in terms of appropriate transition form factors as 


$$
\begin{aligned}
G_{h^{\prime \prime} h^{\prime}}^{\mu}= & \left\langle P^{\prime \prime}, h^{\prime \prime}\left|V^{\mu}\right| P^{\prime}, h^{\prime}\right\rangle \\
= & \varepsilon_{h^{\prime \prime}}^{*} \cdot \varepsilon_{h^{\prime}} P^{\mu} F_{1}\left(q^{2}\right)+\left(\varepsilon_{h^{\prime}}^{\mu} \varepsilon_{h^{\prime \prime}}^{*} \cdot P+\varepsilon_{h^{\prime \prime}}^{* \mu} \varepsilon_{h^{\prime}} \cdot P\right) F_{2}\left(q^{2}\right) \\
& +\frac{1}{2 M^{\prime} M^{\prime \prime}}\left(\varepsilon_{h^{\prime \prime}}^{*} \cdot P\right)\left(\varepsilon_{h^{\prime}} \cdot P\right) P^{\mu} F_{3}\left(q^{2}\right) \\
& +\left(\varepsilon_{h^{\prime}}^{\mu} \varepsilon_{h^{\prime \prime}}^{*} \cdot P-\varepsilon_{h^{\prime \prime}}^{* \mu} \varepsilon_{h^{\prime}} \cdot P\right) F_{4}\left(q^{2}\right) \\
& +\varepsilon_{h^{\prime \prime}}^{*} \cdot \varepsilon_{h^{\prime}} q^{\mu} W_{1}\left(q^{2}\right)+\frac{1}{2 M^{\prime} M^{\prime \prime}}\left(\varepsilon_{h^{\prime \prime}}^{*} \cdot P\right)\left(\varepsilon_{h^{\prime}} \cdot P\right) q^{\mu} W_{2}\left(q^{2}\right),
\end{aligned}
$$

where $P=P^{\prime}+P^{\prime \prime}$ and $q=P^{\prime}-P^{\prime \prime}$. For the special case of an electromagnetic transition $F_{4}\left(q^{2}\right)=W_{i}\left(q^{2}\right)=0$ for $i=1,2$.

We shall always impose the condition $q^{+}=0$, which means that the form factors are known only for spacelike momentum transfer $q^{2}=-q_{\perp}^{2} \leq 0$. In Ref. [17] we have proposed to rewrite a form factor as an explicit function of $q^{2}$ and analytically continue from timelike to spacelike momentum transfer, and verified this procedure in a covariant model calculation in Ref. [8].

The 4-momentum $P^{\prime}$ of a meson of mass $M^{\prime}$ in terms of light-front components is $P^{\prime}=\left(P^{\prime-}, P^{\prime+}, P_{\perp}^{\prime}\right)$, where $P^{\prime \pm}=P^{\prime 0} \pm P^{\prime 3}$ and $P^{\prime 2}=P^{\prime+} P^{\prime-}-P_{\perp}^{\prime 2}=M^{\prime 2}$. The scalar product of two 4 -vectors $\mathrm{A}$ and $\mathrm{B}$ is given by $A \cdot B=\frac{1}{2} A^{+} B^{-}+\frac{1}{2} A^{-} B^{+}-A_{\perp} B_{\perp}$. We use a reference frame, where $q_{\perp}=\left(q^{1}, q^{2}\right)=(Q, 0), P_{\perp}^{\prime}=0$ and $P_{\perp}^{\prime \prime}=-q_{\perp}$. The light-front polarization vector depends upon the momentum of the vector meson. For example, $\varepsilon_{h^{\prime}} \equiv \varepsilon\left(P^{\prime}, h^{\prime}\right)=\left(\varepsilon^{-}, \varepsilon^{+}, \varepsilon_{\perp}\right)$ is given by

$$
\begin{aligned}
\varepsilon\left(P^{\prime}, \pm 1\right) & =\left(\frac{2}{P^{\prime+}} \varepsilon_{\perp}( \pm 1) P_{\perp}^{\prime}, 0, \varepsilon_{\perp}( \pm 1)\right) \\
\varepsilon_{\perp}( \pm 1) & =\frac{\mp 1}{\sqrt{2}}(1, \pm i) \\
\varepsilon\left(P^{\prime}, 0\right) & =\frac{1}{M^{\prime}}\left(\frac{-M^{\prime 2}+P_{\perp}^{\prime 2}}{P^{\prime+}}, P^{\prime+}, P_{\perp}^{\prime}\right),
\end{aligned}
$$

and an analogous expression holds for $\varepsilon_{h^{\prime \prime}}^{*}$.

In this subsection we shall work only with the plus component of the current, and the corresponding matrix element is defined by $G_{h^{\prime \prime} h^{\prime}}^{+}=\omega \cdot G_{h^{\prime \prime} h^{\prime}}$. Since $\omega \cdot q=q^{+}=0$, there are five independent helicity amplitudes which are related to the four form factors, which can be determined in this manner, as

$$
\begin{aligned}
G_{++}^{+} & =-2 M^{\prime}\left(F_{1}+\frac{Q^{2}}{2} \frac{F_{3}}{2 M^{\prime} M^{\prime \prime}}\right), \\
G_{+-}^{+} & =M^{\prime} Q^{2} \frac{F_{3}}{2 M^{\prime} M^{\prime \prime}}, \\
G_{+0}^{+} & =\sqrt{2} Q F_{1}+\frac{Q}{\sqrt{2}}\left(F_{2}-F_{4}\right)+\frac{Q}{\sqrt{2}}\left(Q^{2}-q \cdot P\right) \frac{F_{3}}{2 M^{\prime} M^{\prime \prime}},
\end{aligned}
$$




$$
\begin{aligned}
G_{0+}^{+}= & -\frac{\sqrt{2} M^{\prime} Q}{M^{\prime \prime}} F_{1}-\frac{M^{\prime} Q}{\sqrt{2} M^{\prime \prime}}\left(F_{2}+F_{4}\right)-\frac{M^{\prime} Q}{\sqrt{2} M^{\prime \prime}}\left(Q^{2}+q \cdot P\right) \frac{F_{3}}{2 M^{\prime} M^{\prime \prime}} \\
G_{00}^{+}= & -\frac{1}{M^{\prime \prime}}\left(M^{\prime 2}+M^{\prime \prime 2}-Q^{2}\right) F_{1}+\frac{Q^{2}}{M^{\prime \prime}} F_{2}+\frac{q \cdot P}{M^{\prime \prime}} F_{4} \\
& +\frac{1}{2 M^{\prime \prime}}\left(Q^{4}-(q \cdot P)^{2}\right) \frac{F_{3}}{2 M^{\prime} M^{\prime \prime}}
\end{aligned}
$$

These equations can be solved uniquely for the four form factors only if the helicity amplitudes obey the angular condition

$$
\begin{aligned}
\Delta\left(q^{2}\right) \equiv & G_{00}^{+}-\frac{Q^{2}-q \cdot P}{\sqrt{2} M^{\prime \prime} Q} G_{+0}^{+}+\frac{Q^{2}+q \cdot P}{\sqrt{2} M^{\prime} Q} G_{0+}^{+} \\
& -\frac{M^{\prime 2}+M^{\prime \prime 2}+Q^{2}}{2 M^{\prime} M^{\prime \prime}} G_{++}^{+}-\left(\frac{M^{\prime 2}+M^{\prime \prime 2}}{2 M^{\prime} M^{\prime \prime}}-\frac{3(q \cdot P)^{2}}{2 M^{\prime} M^{\prime \prime} Q^{2}}\right) G_{+-}^{+} \\
= & 0,
\end{aligned}
$$

where $Q^{2}=-q^{2}$. For $q \cdot P=M^{\prime 2}-M^{\prime \prime 2}=0$ and $G_{0+}^{+}=-G_{+0}^{+}$, Eq.(2.9) turns into the usual angular condition $[12,16]$ for electromagnetic transitions.

\subsection{Light-front matrix elements of the electroweak current}

We shall use the same notation as in Ref. [8]. The $q \bar{q}$ meson of mass $M^{\prime}$ and 4-momentum $P^{\prime}$ is composed of off-shell constituent quarks with masses $m_{1}^{\prime}, m_{2}$ and 4-momenta $p_{1}^{\prime}, p_{2}$, respectively, with $p_{1}^{\prime}+p_{2}=P^{\prime}$. The appropriate variables for the internal motion of the constituents, $\left(x, p_{\perp}^{\prime}\right)$, are defined by

$$
\begin{aligned}
& p_{1}^{\prime+}=x P^{\prime+}, \quad p_{2}^{+}=(1-x) P^{\prime+}, \\
& p_{1 \perp}^{\prime}=x P_{\perp}^{\prime}+p_{\perp}^{\prime} \quad, \quad p_{2 \perp}=(1-x) P_{\perp}^{\prime}-p_{\perp}^{\prime},
\end{aligned}
$$

and the kinematic invariant mass is

$$
M_{0}^{\prime 2}=\frac{p_{\perp}^{\prime 2}+m_{1}^{\prime 2}}{x}+\frac{p_{\perp}^{\prime 2}+m_{2}^{2}}{1-x}
$$

The matrix element (2.1) is given in the 1-loop approximation, corresponding to the diagram of Fig.1a, as a light-front integral, which we shall denote by $\hat{G}_{h^{\prime \prime} h^{\prime}}^{\mu}$. For the transition between an initial vector meson with internal variables and masses of its constituent quarks $\left(x, p_{\perp}^{\prime}, m_{1}^{\prime}, m_{2}\right)$ and a final vector meson with the corresponding quantities $\left(x, p_{\perp}^{\prime \prime}, m_{1}^{\prime \prime}, m_{2}\right)$ the momentum integral $\hat{G}_{h^{\prime \prime} h^{\prime}}^{\mu}$ is given by [8]

$$
\hat{G}_{h^{\prime \prime} h^{\prime}}^{\mu}=\frac{N_{c}}{16 \pi^{3}} \int_{0}^{1} d x \int d^{2} p_{\perp}^{\prime} \frac{\varepsilon_{h^{\prime}}^{\beta} t r\left\{\Gamma_{\beta}^{\prime}\left(-\not p_{2}+m_{2}\right) \Gamma_{\alpha}^{\prime \prime}\left(\not p_{1}^{\prime \prime}+m_{1}^{\prime \prime}\right) \gamma^{\mu}\left(\not p_{1}^{\prime}+m_{1}^{\prime}\right)\right\} \varepsilon_{h^{\prime \prime}}^{* \alpha}}{(1-x) \hat{N}_{1}^{\prime} \hat{N}_{1}^{\prime \prime}}
$$

and $N_{c}$ denotes the number of colors. For electromagnetic transitions there is an analoguous contribution due to the diagram of Fig.1b. 


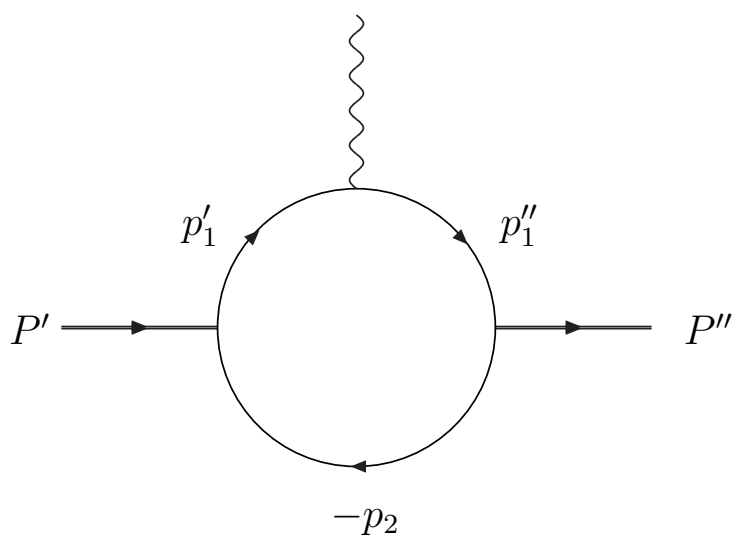

(a)

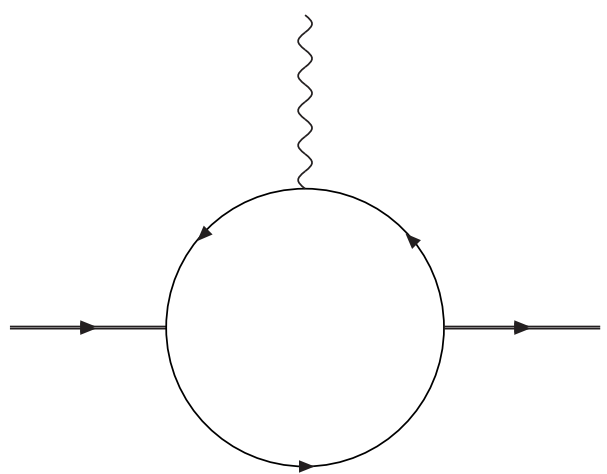

(b)

Figure 1: The one-loop contributions to the transition between $q \bar{q}$ mesons .

Eq.(2.10) is computed at the pole of the spectator quark and we shall use $\hat{p}_{1}^{\prime}, \hat{p}_{1}^{\prime \prime}$ and $\hat{p}_{2}$ to denote the restricted 4 -vectors

$$
\hat{p}_{2}=\left(\frac{m_{2 \perp}^{2}}{p_{2}^{+}}, p_{2}^{+}, p_{2 \perp}\right) \quad, \quad \hat{p}_{1}^{\prime}=P^{\prime}-\hat{p}_{2} \quad, \quad \hat{p}_{1}^{\prime \prime}=\hat{p}_{1}^{\prime}-q,
$$

where $m_{2 \perp}^{2}=p_{2 \perp}^{2}+m_{2}^{2}$. We shall use also the abbreviations $m_{1 \perp}^{\prime 2}=p_{1 \perp}^{\prime 2}+m_{1}^{\prime 2}$ and $m_{1 \perp}^{\prime \prime 2}=p_{1 \perp}^{\prime \prime 2}+m_{1}^{\prime \prime 2}$. In the numerator of the integrand of Eq.(2.10) we have kept the unrestricted constituent momenta for reasons that will be explained below. It follows from Eq. (2.11) that

$$
\begin{aligned}
& \hat{N}_{2}=\hat{p}_{2}^{2}-m_{2}^{2}=0, \\
& \hat{N}_{1}^{\prime}=\hat{p}_{1}^{\prime 2}-m_{1}^{\prime 2}=x\left(M^{\prime 2}-M_{0}^{\prime 2}\right), \\
& \hat{N}_{1}^{\prime \prime}=\hat{p}_{1}^{\prime \prime 2}-m_{1}^{\prime \prime 2}=x\left(M^{\prime \prime 2}-M_{0}^{\prime \prime 2}\right) .
\end{aligned}
$$

The vector vertex operators for ${ }^{3} S_{1}$-state mesons are given in Ref. [8] as

$$
\begin{aligned}
\Gamma_{\beta}^{\prime} \varepsilon_{h^{\prime}}^{\beta} & =-h_{0}^{\prime}\left\{\gamma_{\beta}-\frac{1}{D^{\prime}}\left(p_{1}^{\prime}-p_{2}\right)_{\beta}\right\} \varepsilon_{h^{\prime}}^{\beta}, \\
\Gamma_{\alpha}^{\prime \prime} \varepsilon_{h^{\prime \prime}}^{* \alpha} & =-h_{0}^{\prime \prime}\left\{\gamma_{\alpha}-\frac{1}{D^{\prime \prime}}\left(p_{1}^{\prime \prime}-p_{2}\right)_{\alpha}\right\} \varepsilon_{h^{\prime \prime}}^{* \alpha},
\end{aligned}
$$

where

$$
\begin{aligned}
h_{0}^{\prime} & =\left[\frac{M_{0}^{\prime 4}-\left(m_{1}^{\prime 2}-m_{2}^{2}\right)^{2}}{4 M_{0}^{\prime 3}}\right]^{1 / 2} \frac{M^{\prime 2}-M_{0}^{\prime 2}}{\left[M_{0}^{\prime 2}-\left(m_{1}^{\prime}-m_{2}\right)^{2}\right]^{1 / 2}} \phi\left(M_{0}^{\prime 2}\right), \\
D^{\prime} & =M_{0}^{\prime}+m_{1}^{\prime}+m_{2},
\end{aligned}
$$

and similar equations for $h_{0}^{\prime \prime}$ and $D^{\prime \prime}$. The orbital wave function is assumed to be a simple function of the kinematic invariant mass as

$$
\phi\left(M_{0}^{\prime 2}\right)=Z^{\prime} \exp \left(-M_{0}^{\prime 2} /\left(8 \beta^{\prime 2}\right)\right),
$$


where $Z^{\prime}$ is the normalization constant (see Eq.(4.2)) and the parameter $1 / \beta^{\prime}$ determines the confinement scale.

The helicity amplitude can now be rewritten as

$$
\begin{aligned}
\hat{G}_{h^{\prime \prime} h^{\prime}}^{\mu=} & \frac{N_{c}}{16 \pi^{3}} \int_{0}^{1} d x \int d^{2} p_{\perp}^{\prime} \frac{h_{0}^{\prime} h_{0}^{\prime \prime}}{(1-x) \hat{N}_{1}^{\prime} \hat{N}_{1}^{\prime \prime}} \\
& \left(A^{\mu}-\frac{1}{D^{\prime}} B^{\mu}-\frac{1}{D^{\prime \prime}} C^{\mu}+\frac{1}{D^{\prime} D^{\prime \prime}} D^{\mu}\right),
\end{aligned}
$$

where

$$
\begin{aligned}
A_{\mu} & =\varepsilon_{h^{\prime}}^{\beta} \varepsilon_{h^{\prime \prime}}^{* \alpha} \operatorname{tr}\left[\gamma_{\alpha}\left(\not p_{1}^{\prime \prime}+m_{1}^{\prime \prime}\right) \gamma_{\mu}\left(\not p_{1}^{\prime}+m_{1}^{\prime}\right) \gamma_{\beta}\left(-\not p_{2}+m_{2}\right)\right], \\
B_{\mu} & =\varepsilon_{h^{\prime}} \cdot\left(p_{1}^{\prime}-p_{2}\right) \varepsilon_{h^{\prime \prime}}^{* \alpha} \operatorname{tr}\left[\gamma_{\alpha}\left(\not p_{1}^{\prime \prime}+m_{1}^{\prime \prime}\right) \gamma_{\mu}\left(\not p_{1}^{\prime}+m_{1}^{\prime}\right)\left(-\not p_{2}+m_{2}\right)\right], \\
C_{\mu} & =\varepsilon_{h^{\prime \prime}}^{*} \cdot\left(p_{1}^{\prime \prime}-p_{2}\right) \varepsilon_{h^{\prime}}^{\beta} \operatorname{tr}\left[\left(\not p_{1}^{\prime \prime}+m_{1}^{\prime \prime}\right) \gamma_{\mu}\left(\not p_{1}^{\prime}+m_{1}^{\prime}\right) \gamma_{\beta}\left(-\not p_{2}+m_{2}\right)\right], \\
D_{\mu} & =\varepsilon_{h^{\prime}} \cdot\left(p_{1}^{\prime}-p_{2}\right) \varepsilon_{h^{\prime \prime}}^{*} \cdot\left(p_{1}^{\prime \prime}-p_{2}\right) \operatorname{tr}\left[\left(\not p_{1}^{\prime \prime}+m_{1}^{\prime \prime}\right) \gamma_{\mu}\left(\not p_{1}^{\prime}+m_{1}^{\prime}\right)\left(-\not p_{2}+m_{2}\right)\right] .
\end{aligned}
$$

With $p_{2}=P^{\prime}-p_{1}^{\prime}$ and $p_{1}^{\prime \prime}=p_{1}^{\prime}-q$ the traces of Eq.(2.18) can be expressed in terms of the tensors $p_{1 \mu}^{\prime}, p_{1 \mu}^{\prime} p_{1 \nu}^{\prime}$ and $p_{1 \mu}^{\prime} p_{1 \nu}^{\prime} p_{1 \alpha}^{\prime}$, whose decompositions into the 4vectors $P, q$ and $\omega$ are given in Appendix A. Special care is taken to trace the $p_{1}^{\prime-}$ dependence, which is encoded in the functions $B_{n}^{(m)}$ and $C_{n}^{(m)}$. We emphasize that only the decomposition coefficients which are combined with the 4-vector $\omega$, namely $B_{n}^{(m)}$ and $C_{n}^{(m)}$, depend on $p_{1}^{\prime-}$ and behave like $\left(p_{1}^{\prime-}\right)^{i}\left(p_{1}^{\prime+}\right)^{j}$. For the $B$-functions $i \leq j$, which means that there is no zero mode contribution and the value of $B_{n}^{(m)}$ can be calculated unambiguously at the spectator quark pole, Eq.(2.11). For the C-functions $i \geq j+1$, and the value of $C_{n}^{(m)}$ is the sum of a spectator quark pole term and an unknown zero mode contribution, as we have shown in a model calculation in Ref. [8].

Note that if the integral $(2.17)$ is calculated at the pole of the spectator quark it is known exactly: the minus components of the internal momenta are given by Eq.(2.11), in particular $p_{1}^{\prime-}=\hat{p}_{1}^{--}=P^{\prime-}-m_{2 \perp}^{2} / p_{2}^{+}$, and the $C$-functions can be evaluated in this case. However, such a prescription misses entirely the effect of zero modes and violates rotational invariance, i.e., is not Lorentz covariant. Full covariance is restored by the zero mode terms of the $C$-functions.

Another prescription to fix the minus components of the constituents is used in the standard light-front formalism (and the CDKM scheme), where all quarks are put on their respective mass shells, i.e., $p_{1}^{\prime-}=m_{1 \perp}^{\prime 2} / p_{1}^{\prime+}, p_{1}^{\prime \prime-}=m_{1 \perp}^{\prime \prime 2} / p_{1}^{\prime \prime+}$ and $p_{2}^{-}=m_{2 \perp}^{2} / p_{2}^{+}$. This is the prescription used most often in the literature. The resulting light-front integral is known exactly, but it contains spurious contributions due to unresolved zero mode effects.

In our approach we shall not fix the value of $p_{1}^{--}$and shall use the $C$-functions as a convenient parametrization for the contribution of zero modes. But it is not known as yet how to calculate the contribution of the zero modes associated with a light-front integral like (2.17). While various model calculations $[8,9,10,11]$ have 
been used to investigate the role of zero modes in a light-front approach, they did not provide a practical method for a realistic phenomenology. We shall address this problem in the present work.

Consequently, the light-front integral (2.17) must be represented also in terms of the 4-vectors $P, q$ and $\omega$ :

$$
\begin{aligned}
\hat{G}_{h^{\prime \prime} h^{\prime}}^{\mu}= & \varepsilon_{h^{\prime \prime}}^{*} \cdot \varepsilon_{h^{\prime}} P^{\mu} F_{1}\left(q^{2}\right)+\left(\varepsilon_{h^{\prime}}^{\mu} \varepsilon_{h^{\prime \prime}}^{*} \cdot P+\varepsilon_{h^{\prime \prime}}^{* \mu} \varepsilon_{h^{\prime}} \cdot P\right) F_{2}\left(q^{2}\right) \\
& +\frac{1}{2 M^{\prime} M^{\prime \prime}}\left(\varepsilon_{h^{\prime \prime}}^{*} \cdot P\right)\left(\varepsilon_{h^{\prime}} \cdot P\right) P^{\mu} F_{3}\left(q^{2}\right) \\
& +\left(\varepsilon_{h^{\prime}}^{\mu} \varepsilon_{h^{\prime \prime}}^{*} \cdot P-\varepsilon_{h^{\prime \prime}}^{* \mu} \varepsilon_{h^{\prime}} \cdot P\right) F_{4}\left(q^{2}\right) \\
& +\frac{1}{\omega \cdot P}\left(\varepsilon_{h^{\prime}}^{\mu} \varepsilon_{h^{\prime \prime}}^{*} \cdot \omega+\varepsilon_{h^{\prime \prime}}^{* \mu} \varepsilon_{h^{\prime}} \cdot \omega\right) H_{1}\left(q^{2}\right) \\
& +\frac{1}{\omega \cdot P}\left(\varepsilon_{h^{\prime}}^{\mu} \varepsilon_{h^{\prime \prime}}^{*} \cdot \omega-\varepsilon_{h^{\prime \prime}}^{* \mu} \varepsilon_{h^{\prime}} \cdot \omega\right) H_{2}\left(q^{2}\right) \\
& +q^{\mu}(\cdots)+\omega^{\mu}(\cdots),
\end{aligned}
$$

where we have omitted all terms proportional to $q^{\mu}$ and $\omega^{\mu}$, and we have used the same notation for the form factors $F_{i}$ regardless if they represent the approximation (2.17) or the exact helicity amplitude (2.1). The approximate helicity amplitude $\hat{G}_{h^{\prime \prime} h^{\prime}}^{\mu}$ consists of an $\omega$ independent part, and a part that does depend on $\omega$ and whose construction requires additional, unphysical form factors. However, this separation is not unique. For example, in writing down Eq.(2.19) we have used the identity

$$
\begin{aligned}
\frac{1}{\omega \cdot P} P^{\mu} \varepsilon_{h} \cdot \omega= & \varepsilon_{h}^{\mu}-\frac{q^{\mu}}{q^{2}}\left(\varepsilon_{h} \cdot q-q \cdot P \frac{\varepsilon_{h} \cdot \omega}{\omega \cdot P}\right) \\
& -\frac{\omega^{\mu}}{\omega \cdot P}\left(\varepsilon_{h} \cdot P-\varepsilon_{h} \cdot q \frac{q \cdot P}{q^{2}}-\varepsilon_{h} \cdot \omega \frac{P^{2}}{\omega \cdot P}\right. \\
& \left.+\varepsilon_{h} \cdot \omega \frac{(q \cdot P)^{2}}{q^{2} \omega \cdot P}\right) \\
& -\frac{1}{q^{2}(\omega \cdot P)^{2}} \varepsilon^{\mu \alpha \beta \nu} \omega_{\alpha} q_{\beta} P_{\nu} \varepsilon_{\rho \tau \sigma \gamma} \varepsilon_{h}^{\rho} \omega^{\tau} q^{\sigma} P^{\gamma}
\end{aligned}
$$

in order to absorb the contributions due to the terms proportional to

$$
\frac{1}{\omega \cdot P} P_{\mu} \varepsilon_{h^{\prime}} \cdot \omega \varepsilon_{h^{\prime \prime}}^{*} \cdot P, \frac{1}{\omega \cdot P} P_{\mu} \varepsilon_{h^{\prime \prime}}^{*} \cdot \omega \varepsilon_{h^{\prime}} \cdot P, \frac{1}{(\omega \cdot P)^{2}} P_{\mu} \varepsilon_{h^{\prime}} \cdot \omega \varepsilon_{h^{\prime \prime}}^{*} \cdot \omega,
$$

into the form factors $F_{2}, F_{4}, H_{1}$ and $H_{2}$.

We have made no reference to the $\varepsilon$-tensor terms in Eq.(2.19) and shall treat them as unphysical contributions which can be neglected. This procedure is well justified for the longitutinal mode since the $\varepsilon$-tensor term vanishes for $h=0$, because

$$
\frac{1}{\omega \cdot P} \varepsilon_{\rho \tau \sigma \gamma} \varepsilon_{h}^{\rho} \omega^{\tau} q^{\sigma} P^{\gamma}=i h \varepsilon_{h} \cdot q
$$


For the transverse mode the decomposition into physical and unphysical contributions, resulting from the identity (2.20), is ambiguous, but is consistent with the result obtained for the longitutinal mode. In particular, the relationship between the four form factors $F_{i}\left(q^{2}\right)$ and the plus components of the helicity amplitudes $\hat{G}_{h^{\prime \prime} h^{\prime}}^{+}$ agree exactly with Eqs.(2.4)-(2.7), which means that

$$
\hat{G}_{++}^{+}=G_{++}^{+}, \hat{G}_{+-}^{+}=G_{+-}^{+}, \hat{G}_{+0}^{+}=G_{+0}^{+}, \hat{G}_{0+}^{+}=G_{0+}^{+},
$$

where the difference between the exact and the approximate helicity amplitude is understood. Only the amplitude $\hat{G}_{00}^{+}$depends on one of the unphysical form factors in the following way:

$$
\hat{G}_{00}^{+}=G_{00}^{+}+\frac{1}{M^{\prime \prime}} H_{1} .
$$

It is therefore not surprising that the angular condition (2.9) requires the unphysical form factor $H_{1}$ to vanish:

$$
\Delta\left(q^{2}\right)=\frac{1}{M^{\prime \prime}} H_{1}\left(q^{2}\right)=0 .
$$

The determination of the light-front integrals for the form factors $F_{i}\left(q^{2}\right)$ is straightforward; it can be derived from the detailed decomposition of the traces (2.18), which is exhibited for the special case of an electromagnetic transition in Appendix B. Note that the expressions for $F_{2}$ (and $F_{4}$ ) contain $C$-functions, which means that both form factors are affected by zero modes.

Only the amplitude $\hat{G}_{00}^{+}$contains admixtures of unphysical form factors, which confirms the result of Ref. [16] that the light-front analysis that uses the plus components of the helicity amplitudes, avoiding the $\left(h^{\prime \prime}, h^{\prime}\right)=(0,0)$ component, is preferred for model calculations. The form factors $F_{i}$ derived according to this prescription agree exactly with our results. However, the zero mode problem cannot be avoided since, as noted above, the calculation that uses the vertex operators (2.13) leads to form factors that require zero mode contributions (these are related to the second term of the vertex operator).

In the CDKM scheme of Refs. $[2,18]$ the helicity amplitude is represented also in terms of the 4 -vectors $P, q$ and $\omega$, and the $\omega$-dependent contributions are separated and omitted entirely. But this approach has the same problems: a unique separation is not possible, and the form factor $F_{2}$ is in general affected by zero modes.

We shall now discuss the form factor $H_{1}\left(q^{2}\right)$ in greater detail. It can be represented in terms of the functions $B_{n}^{(m)}$ and $C_{n}^{(m)}$, which have been defined in Appendix A. According to the representation used in Eq.(2.17) one finds

$$
H_{1}\left(q^{2}\right)=H_{1}^{A}+H_{1}^{B}+H_{1}^{C}+H_{1}^{D}
$$

with

$$
H_{1}^{A}\left(q^{2}\right)=\frac{N_{c}}{16 \pi^{3}} \int_{0}^{1} d x \int d^{2} p_{\perp}^{\prime} \frac{h_{0}^{\prime} h_{0}^{\prime \prime}}{(1-x) \hat{N}_{1}^{\prime} \hat{N}_{1}^{\prime \prime}}(-4)\left(Y-m_{1}^{\prime} m_{1}^{\prime \prime} C_{1}^{(1)}\right)
$$


where

$$
Y=2 C_{1}^{(3)}+q^{2}\left(C_{2}^{(3)}-C_{1}^{(2)}\right)+P^{2} B_{1}^{(3)}+2 q \cdot P B_{2}^{(3)}-q \cdot P B_{1}^{(2)} .
$$

For $H_{1}^{B}, H_{1}^{C}$ and $H_{1}^{D}$ one finds

$$
\begin{aligned}
H_{1}^{B}\left(q^{2}\right)= & \frac{N_{c}}{16 \pi^{3}} \int_{0}^{1} d x \int d^{2} p_{\perp}^{\prime} \frac{h_{0}^{\prime} h_{0}^{\prime \prime}}{(1-x) \hat{N}_{1}^{\prime} \hat{N}_{1}^{\prime \prime}} \\
& \times \frac{-2}{D^{\prime}}\left\{-2\left(m_{1}^{\prime}+m_{2}\right)\left[Y-m_{1}^{\prime} m_{1}^{\prime \prime} C_{1}^{(1)}\right]+2 m_{1}^{\prime \prime} C_{1}^{(1)} N_{1}^{\prime}\right. \\
& \quad+\left(m_{1}^{\prime}-m_{1}^{\prime \prime}\right)\left[\left(q \cdot P+q^{2}\right) C_{1}^{(2)}+\left(P^{2}+q \cdot P\right) B_{1}^{(2)}\right] \\
& \left.-\left(q^{2}+q \cdot P\right) m_{1}^{\prime} C_{1}^{(1)}\right\} \\
H_{1}^{C}\left(q^{2}\right)= & \frac{N_{c}}{16 \pi^{3}} \int_{0}^{1} d x \int d^{2} p_{\perp}^{\prime} \frac{h_{0}^{\prime} h_{0}^{\prime \prime}}{(1-x) \hat{N}_{1}^{\prime} \hat{N}_{1}^{\prime \prime}} \\
& \times \frac{-2}{D^{\prime \prime}}\left\{-2\left(m_{1}^{\prime \prime}+m_{2}\right)\left[Y-m_{1}^{\prime} m_{1}^{\prime \prime} C_{1}^{(1)}\right]+2 m_{1}^{\prime} C_{1}^{(1)} N_{1}^{\prime \prime}\right. \\
& \quad-\left(m_{1}^{\prime}-m_{1}^{\prime \prime}\right)\left[\left(q \cdot P-q^{2}\right) C_{1}^{(2)}+\left(P^{2}-q \cdot P\right) B_{1}^{(2)}\right] \\
& \left.\quad q \cdot P) m_{1}^{\prime} C_{1}^{(1)}\right\}, \\
H_{1}^{D}\left(q^{2}\right)=\frac{N_{c}}{16 \pi^{3}} \int_{0}^{1} d x \int d^{2} p_{\perp}^{\prime} \frac{h_{0}^{\prime} h_{0}^{\prime \prime}}{(1-x) \hat{N}_{1}^{\prime} \hat{N}_{1}^{\prime \prime}} & \quad \frac{4}{D^{\prime} D^{\prime \prime}}\left\{\left[q^{2}-M^{\prime 2}-M^{\prime \prime 2}+2 N_{2}-\left(m_{1}^{\prime}-m_{1}^{\prime \prime}\right)^{2}+\left(m_{1}^{\prime}+m_{2}\right)^{2}\right.\right. \\
& \left.\quad+\left(m_{1}^{\prime \prime}+m_{2}\right)^{2}\right]\left(2 C_{1}^{(3)}+C_{3}^{(3)}\right) \\
& \left.\quad \frac{1}{2}\left[q^{2}-N_{1}^{\prime}-N_{1}^{\prime \prime}-\left(m_{1}^{\prime}-m_{1}^{\prime \prime}\right)^{2}\right] C_{2}^{(2)}\right\} \cdot
\end{aligned}
$$

\subsection{Determination of effective zero mode contributions}

Obviously, the angular condition (2.23) constrains a combination of $C$-functions and consequently contains information on the effect of zero modes. In order to exploit this hint in more detail, we use the fact (compare for example Refs. $[19,8]$ ) that the light-front integral (2.10) can be derived, in principle, from an explicitly covariant, 4-dimensional momentum integral: The latter is expressed in terms of light-front variables and carried out by contour methods in the complex $p_{1}^{\prime-}$ plane. Closing the contour in the upper $p_{1}^{\prime-}$ plane (it is assumed that the vertex functions $h_{0}^{\prime}$ and $h_{0}^{\prime \prime}$ have no poles there) ensures that the momentum integral is given by the residue of the spectator quark pole, corresponding to putting quark 2 on the mass

shell. The resulting residue coincides with the light-front integral (2.10) with the prescription (2.11). If the light-front integral could be completed by the associated 
zero mode contributions, the result would be equivalent to the original, covariant 1loop integral. We have argued that the zero modes are related to the $p_{1}^{\prime-}$ dependent terms of the traces in the representation (2.17) of the light-front integral, and we have encoded this $p_{1}^{\prime-}$ dependence in terms of $C$-functions. The requirement of covariance then leads to the angular condition $(2.23)$.

However, each of the four trace terms $A_{\mu}, B_{\mu}, C_{\mu}$ and $D_{\mu}$ corresponds separately to a covariant, 4-dimensional structure in the sense explained above. Each individual contribution to the light-front integral (2.17) must be consistent with the requirement of covariance, which immediately leads to the conditions

$$
H_{1}^{A}=H_{1}^{B}=H_{1}^{C}=H_{1}^{D}=0,
$$

which, of course, are consistent with the angular condition (2.23).

We can go further and use also the facts, that the zero mode contributions do not depend on the details of the spinor structure of the vertex operators, and that different combinations of vertex spin structures may generate the same formal decomposition (2.19) of the light-front integral. For example, we can formally replace the trace $A_{\mu} \equiv A_{\mu}(V V V)$, Eq.(2.18), by three different expressions:

$$
\begin{aligned}
A_{\mu}(A V A) & =\varepsilon_{h^{\prime}}^{\beta} \varepsilon_{h^{\prime \prime}}^{* \alpha} \operatorname{tr}\left[\gamma_{\alpha} \gamma_{5}\left(\not p_{1}^{\prime \prime}+m_{1}^{\prime \prime}\right) \gamma_{\mu}\left(\not p_{1}^{\prime}+m_{1}^{\prime}\right) \gamma_{\beta} \gamma_{5}\left(-\not p_{2}+m_{2}\right)\right] \\
& =\left.A_{\mu}(V V V)\right|_{m_{2} \rightarrow-m_{2}}, \\
A_{\mu}(V A A) & =\varepsilon_{h^{\prime}}^{\beta} \varepsilon_{h^{\prime \prime}}^{* \alpha} \operatorname{tr}\left[\gamma_{\alpha} \gamma_{5}\left(\not p_{1}^{\prime \prime}+m_{1}^{\prime \prime}\right) \gamma_{\mu} \gamma_{5}\left(\not p_{1}^{\prime}+m_{1}^{\prime}\right) \gamma_{\beta}\left(-\not p_{2}+m_{2}\right)\right] \\
& =\left.A_{\mu}(V V V)\right|_{m_{1}^{\prime} \rightarrow-m_{1}^{\prime}}, \\
A_{\mu}(A A V) & =\varepsilon_{h^{\prime}}^{\beta} \varepsilon_{h^{\prime \prime}}^{* \alpha} \operatorname{tr}\left[\gamma_{\alpha}\left(\not p_{1}^{\prime \prime}+m_{1}^{\prime \prime}\right) \gamma_{\mu} \gamma_{5}\left(\not \not p_{1}^{\prime}+m_{1}^{\prime}\right) \gamma_{\beta} \gamma_{5}\left(-\not p_{2}+m_{2}\right)\right] \\
& =\left.A_{\mu}(V V V)\right|_{m_{1}^{\prime \prime} \rightarrow-m_{1}^{\prime \prime}} .
\end{aligned}
$$

Obviously, the traces $A_{\mu}(A V A), A_{\mu}(V A A)$ and $A_{\mu}(A A V)$ are obtained from $A_{\mu}(V V V)$ by changing the signs of $m_{2}, m_{1}^{\prime}$ and $m_{1}^{\prime \prime}$, respectively, while the $p_{1}^{\prime-}$ dependent terms remain unchanged. There are corresponding consistency conditions that can be derived from $H_{1}^{A} \equiv H_{1}^{A}(V V V)=0$, Eq.(2.30), by the analogous change of the sign of one of the quark masses. In this manner one finds that

$$
H_{1}^{A}(A V A)=H_{1}^{A}(V A A)=H_{1}^{A}(V A A)=0 .
$$

The same procedure can be applied to the traces $B_{\mu}, C_{\mu}$ and $D_{\mu}$ with analoguous results (modulo an irrelevant overall change of sign). Consequently, the consistency conditions (2.30) are invariant under the change of sign of one of the quark masses in that part of the light-front integral which depends on one of the traces (2.18).

Since the functions $B_{n}^{(m)}$ and $C_{n}^{(m)}$ depend only on the squared quark masses, this sign invariance of the consistency condition $H_{1}^{A}=0$ means, that the two terms of Eq.( 2.25) must vanish separately:

$$
\begin{aligned}
\int_{0}^{1} d x \int d^{2} p_{\perp}^{\prime} \frac{h_{0}^{\prime} h_{0}^{\prime \prime}}{(1-x) \hat{N}_{1}^{\prime} \hat{N}_{1}^{\prime \prime}} Y & =0, \\
\int_{0}^{1} d x \int d^{2} p_{\perp}^{\prime} \frac{h_{0}^{\prime} h_{0}^{\prime \prime}}{(1-x) \hat{N}_{1}^{\prime} \hat{N}_{1}^{\prime \prime}} C_{1}^{(1)} & =0 .
\end{aligned}
$$


Similarly, the sign invariance of the consistency conditions $H_{1}^{B}=0$ and $H_{1}^{C}=0$, Eqs.(2.27), (2.28), leads to the relations:

$$
\begin{gathered}
\int_{0}^{1} d x \int d^{2} p_{\perp}^{\prime} \frac{h_{0}^{\prime} h_{0}^{\prime \prime}}{(1-x) \hat{N}_{1}^{\prime} \hat{N}_{1}^{\prime \prime}} \frac{1}{D^{\prime}} Y \\
=\int_{0}^{1} d x \int d^{2} p_{\perp}^{\prime} \frac{h_{0}^{\prime} h_{0}^{\prime \prime}}{(1-x) \hat{N}_{1}^{\prime} \hat{N}_{1}^{\prime \prime}} \frac{1}{D^{\prime \prime}} Y=0 \\
\int_{0}^{1} d x \int d^{2} p_{\perp}^{\prime} \frac{h_{0}^{\prime} h_{0}^{\prime \prime}}{(1-x) \hat{N}_{1}^{\prime} \hat{N}_{1}^{\prime \prime}} \frac{1}{D^{\prime}} C_{1}^{(1)} \\
=\int_{0}^{1} d x \int d^{2} p_{\perp}^{\prime} \frac{h_{0}^{\prime} h_{0}^{\prime \prime}}{(1-x) \hat{N}_{1}^{\prime} \hat{N}_{1}^{\prime \prime}} \frac{1}{D^{\prime \prime}} C_{1}^{(1)}=0 \\
\int_{0}^{1} d x \int d^{2} p_{\perp}^{\prime} \frac{h_{0}^{\prime} h_{0}^{\prime \prime}}{(1-x) \hat{N}_{1}^{\prime} \hat{N}_{1}^{\prime \prime}} \frac{1}{D^{\prime}} N_{1}^{\prime} C_{1}^{(1)} \\
=\int_{0}^{1} d x \int d^{2} p_{\perp}^{\prime} \frac{h_{0}^{\prime} h_{0}^{\prime \prime}}{(1-x) \hat{N}_{1}^{\prime} \hat{N}_{1}^{\prime \prime}} \frac{1}{D^{\prime \prime}} N_{1}^{\prime \prime} C_{1}^{(1)}=0 \\
\int_{0}^{1} d x \int d^{2} p_{\perp}^{\prime} \frac{h_{0}^{\prime} h_{0}^{\prime \prime}}{(1-x) \hat{N}_{1}^{\prime} \hat{N}_{1}^{\prime \prime}} \frac{1}{D^{\prime}}\left[\left(q \cdot P+q^{2}\right) C_{1}^{(2)}\right. \\
\left.+\left(P^{2}+q \cdot P\right) B_{1}^{(2)}\right] \\
=\int_{0}^{1} d x \int d^{2} p_{\perp}^{\prime} \frac{h_{0}^{\prime} h_{0}^{\prime \prime}}{(1-x) \hat{N}_{1}^{\prime} \hat{N}_{1}^{\prime \prime}} \frac{1}{D^{\prime \prime}}\left[\left(q \cdot P-q^{2}\right) C_{1}^{(2)}+\left(P^{2}-q \cdot P\right) B_{1}^{(2)}\right]=0 .
\end{gathered}
$$

We shall not consider the relations that can be derived from the consistency condition $H_{1}^{D}=0$, but we shall assume that

$$
\begin{aligned}
& \int_{0}^{1} d x \int d^{2} p_{\perp}^{\prime} \frac{h_{0}^{\prime} h_{0}^{\prime \prime}}{(1-x) \hat{N}_{1}^{\prime} \hat{N}_{1}^{\prime \prime}} \frac{1}{D^{\prime} D^{\prime \prime}} Y=0, \\
& \int_{0}^{1} d x \int d^{2} p_{\perp}^{\prime} \frac{h_{0}^{\prime} h_{0}^{\prime \prime}}{(1-x) \hat{N}_{1}^{\prime} \hat{N}_{1}^{\prime \prime}} \frac{1}{D^{\prime} D^{\prime \prime}} C_{1}^{(1)}=0 .
\end{aligned}
$$

These relations cannot be derived directly but they are a natural generalization of Eqs.(2.35)-(2.38).

The consistency relations (2.35)-(2.42) can be interpreted as an account of the effect of zero modes in terms of light-front integrals. These results are very useful for practical calculations. For example, the functions $C_{1}^{(1)}, Y$ and $C_{1}^{(2)}$ contained in the form factor $F_{2}\left(q^{2}\right)$ can be determined by means of Eqs.(2.38) and (2.40)-(2.42). Consequently, the form factors $F_{i}\left(q^{2}\right)$ can now be predicted uniquely. 


\section{Calculation of form factors that require zero mode contributions}

There is one class of form factors like the electromagnetic form factor of a pseudoscalar meson, $V$ and $A_{2}$ for transitions between vector and pseudoscalar mesons, and the pseudoscalar coupling constant $f_{P}$ that are free of $C$-functions. These quantities are predicted unambiguously in 1-loop order by the standard light-front approach. Another class of form factors like $A_{1}$ and the vector coupling constant $f_{V}$ depend on $C$-functions. For their reliable prediction in 1-loop order the associated zero mode contribution must be known. Fortunately, there are only two combinations of $C$-functions, namely $C_{1}^{(1)}$ and $Y$, that are common to all $C$-dependent form factors considered in this work. Therefore, the consistency relations (2.35)-(2.42) for the functions $C_{1}^{(1)}$ and $Y$, which we have derived for transitions between $q \bar{q}$ vector mesons, can be used also in other processes to determine uniquely those form factors that are affected by zero modes. We shall calculate $A_{1}\left(q^{2}\right)$ and $f_{V}$ in this manner; the corresponding formulas in the SLF and CDKM schemes will be derived in Appendix C.

\subsection{The axial form factor $A_{1}\left(q^{2}\right)$ for transitions between pseudoscalar and vector mesons}

We shall consider the matrix element of the axial vector current $A^{\mu}=\bar{q}^{\prime \prime} \gamma^{\mu} \gamma_{5} q^{\prime}$ between an initial pseudoscalar and a final vector meson state of 4-momentum, mass and helicity $\left(P^{\prime}, M^{\prime}\right)$ and $\left(P^{\prime \prime}, M^{\prime \prime}, h^{\prime \prime}\right)$, respectively. Using the same notation as in Eq.(2.1) it is represented as

$$
G_{h^{\prime \prime}}^{\mu}=\left\langle P^{\prime \prime}, h^{\prime \prime}\left|A^{\mu}\right| P^{\prime}\right\rangle=\varepsilon_{h^{\prime \prime}}^{* \mu} f\left(q^{2}\right)+P^{\mu} \varepsilon_{h^{\prime \prime}}^{*} \cdot P a_{+}\left(q^{2}\right)+q^{\mu} \varepsilon_{h^{\prime \prime}}^{*} \cdot P a_{-}\left(q^{2}\right),
$$

where the form factors defined in Eq.(3.1) are related to the convention used most frequently by

$$
\begin{aligned}
& A_{1}\left(q^{2}\right)=-\left(M^{\prime}+M^{\prime \prime}\right)^{-1} f\left(q^{2}\right) \\
& A_{2}\left(q^{2}\right)=\left(M^{\prime}+M^{\prime \prime}\right) a_{+}\left(q^{2}\right) .
\end{aligned}
$$

This matrix element is given in the 1-loop approximation, corresponding to the diagram of Fig.1a, as a light-front integral, which we shall denote by $\hat{G}_{h^{\prime \prime}}^{\mu}$

$$
\hat{G}_{h^{\prime \prime}}^{\mu}=\frac{N_{c}}{16 \pi^{3}} \int_{0}^{1} d x \int d^{2} p_{\perp}^{\prime} \frac{\varepsilon_{h^{\prime \prime}}^{* \alpha} \operatorname{tr}\left\{\Gamma^{\prime}\left(-\not p_{2}+m_{2}\right) \Gamma_{\alpha}^{\prime \prime}\left(\not p_{1}^{\prime \prime}+m_{1}^{\prime \prime}\right) \gamma^{\mu} \gamma_{5}\left(\not p_{1}^{\prime}+m_{1}^{\prime}\right)\right\}}{(1-x) \hat{N}_{1}^{\prime} \hat{N}_{1}^{\prime \prime}}
$$

The pseudoscalar vertex operator for a ${ }^{1} S_{0}$-state meson is [8]

$$
\Gamma^{\prime}=h_{0}^{\prime} \gamma_{5}
$$


and with the vector vertex operator $(2.13)$ the amplitude $\hat{G}_{h^{\prime \prime}}^{\mu}$ can be rewritten as

$$
\hat{G}_{h^{\prime \prime}}^{\mu}=\frac{N_{c}}{16 \pi^{3}} \int_{0}^{1} d x \int d^{2} p_{\perp}^{\prime} \frac{h_{0}^{\prime} h_{0}^{\prime \prime}}{(1-x) \hat{N}_{1}^{\prime} \hat{N}_{1}^{\prime \prime}}\left(\hat{A}^{\mu}+\frac{1}{D^{\prime \prime}} \hat{B}^{\mu}\right)
$$

where

$$
\begin{aligned}
& \hat{A}_{\mu}=-\varepsilon_{h^{\prime \prime}}^{* \alpha} \operatorname{tr}\left[\gamma_{\alpha}\left(\not p_{1}^{\prime \prime}+m_{1}^{\prime \prime}\right) \gamma_{\mu} \gamma_{5}\left(\not p_{1}^{\prime}+m_{1}^{\prime}\right) \gamma_{5}\left(-\not p_{2}+m_{2}\right)\right] \\
& \hat{B}_{\mu}=\varepsilon_{h^{\prime \prime}}^{*} \cdot\left(p_{1}^{\prime \prime}-p_{2}\right) \operatorname{tr}\left[\left(\not p_{1}^{\prime \prime}+m_{1}^{\prime \prime}\right) \gamma_{\mu} \gamma_{5}\left(\not p_{1}^{\prime}+m_{1}^{\prime}\right) \gamma_{5}\left(-\not p_{2}+m_{2}\right)\right] .
\end{aligned}
$$

Based upon the tensor decomposition of Appendix A the traces have been represented in Appendix C; the result for the light-front integral is now given by

$$
\hat{G}_{h^{\prime \prime}}^{\mu}=\varepsilon_{h^{\prime \prime}}^{* \mu} f\left(q^{2}\right)+P^{\mu} \varepsilon_{h^{\prime \prime}}^{*} \cdot P a_{+}\left(q^{2}\right)+q^{\mu}(\cdots)+\omega^{\mu}(\cdots) .
$$

We have again used the identity (2.20) to absorb the contribution due to the term $\frac{1}{\omega \cdot P} P^{\mu} \varepsilon_{h^{\prime \prime}}^{*} \cdot \omega$ into the form factor $f\left(q^{2}\right)$ and have omitted the $\varepsilon$-tensor term in Eq.(3.7). The form factor $a_{+}\left(q^{2}\right)$ is free of zero mode contributions and we copy from Ref. [19]:

$$
\begin{aligned}
a_{+}\left(q^{2}\right)= & \frac{N_{c}}{16 \pi^{3}} \int_{0}^{1} d x \int d^{2} p_{\perp}^{\prime} \frac{2 h_{0}^{\prime} h_{0}^{\prime \prime}}{(1-x) \hat{N}_{1}^{\prime} \hat{N}_{1}^{\prime \prime}}\left\{(2 x-1)\left[(1-x) m_{1}^{\prime}+x m_{2}\right]\right. \\
& -\left[2 x m_{2}+m_{1}^{\prime \prime}+(1-2 x) m_{1}^{\prime}\right] \frac{p_{\perp}^{\prime} q_{\perp}}{q^{2}} \\
& \left.-2 \frac{(1-x) q^{2}+p_{\perp}^{\prime} q_{\perp}}{(1-x) q^{2} D^{\prime \prime}}\left(p_{\perp}^{\prime} p_{\perp}^{\prime \prime}+\left[x m_{2}+(1-x) m_{1}^{\prime}\right]\left[x m_{2}-(1-x) m_{1}^{\prime \prime}\right]\right)\right\},
\end{aligned}
$$

while $f\left(q^{2}\right)$ is

$$
\begin{aligned}
f\left(q^{2}\right)= & \frac{N_{c}}{16 \pi^{3}} \int_{0}^{1} d x \int d^{2} p_{\perp}^{\prime} \frac{h_{0}^{\prime} h_{0}^{\prime \prime}}{(1-x) \hat{N}_{1}^{\prime} \hat{N}_{1}^{\prime \prime}}\left\{2 x\left(m_{2}-m_{1}^{\prime}\right)\left(M_{0}^{\prime 2}+M_{0}^{\prime 2}\right)-4 x m_{1}^{\prime \prime} M_{0}^{\prime 2}\right. \\
& +2(1-x) m_{1}^{\prime} q \cdot P+2 m_{2} q^{2}-2 x m_{2}\left(M^{\prime 2}+M^{\prime \prime 2}\right) \\
& +2\left(m_{1}^{\prime}-m_{2}\right)\left(m_{1}^{\prime}+m_{1}^{\prime \prime}\right)^{2} \\
& -8\left(m_{1}^{\prime}-m_{2}\right) A_{1}^{(2)}+2\left(m_{1}^{\prime}+m_{1}^{\prime \prime}\right)\left(q^{2}+q \cdot P\right) \frac{p_{\perp}^{\prime} q_{\perp}}{q^{2}}-8\left(m_{1}^{\prime}-m_{2}\right) B_{1}^{(2)} \\
& +\frac{4}{D^{\prime \prime}}\left[A _ { 1 } ^ { ( 2 ) } \left(2 x M^{\prime 2}+2 x M_{0}^{\prime 2}-q^{2}-q \cdot P-2\left(q^{2}+q \cdot P\right) \frac{p_{\perp}^{\prime} q_{\perp}}{q^{2}}\right.\right. \\
& \left.-2\left(m_{1}^{\prime}-m_{1}^{\prime \prime}\right)\left(m_{1}^{\prime}-m_{2}\right)\right) \\
& -2 B_{3}^{(3)}+\left(M^{\prime 2}+M^{\prime \prime 2}-q^{2}+2\left(m_{1}^{\prime}-m_{2}\right)\left(m_{1}^{\prime \prime}+m_{2}\right)\right) B_{1}^{(2)} \\
& \left.\left.-m_{1}^{\prime} m_{1}^{\prime \prime} C_{1}^{(1)}-Y\right]\right\},
\end{aligned}
$$


where $A_{1}^{(2)}, B_{1}^{(2)}$ and $C_{1}^{(1)}$ are defined in Appendix A. Note that exactly the same result for $f\left(q^{2}\right)$ can be derived from the plus component of Eq.(3.5), for the longitutinal decay mode, $\hat{G}_{0}^{+}$, without using the decomposition (3.7).

The functions $C_{1}^{(1)}$ and $Y$, Eq.(2.26), are associated with zero mode effects, and it is remarkable that there appear the same groups of $C$-functions in Eq.(3.9) as in the consistency relations of the previous section. In particular, Eqs.(2.37) and (2.38) can be used to determine the values of $C_{1}^{(1)}$ and $Y$ in Eq.(3.9):

$$
C_{1}^{(1)} \doteq 0 \quad \text { and } \quad Y \doteq 0
$$

The unique result for the form factor $f\left(q^{2}\right)$ is given by Eqs.(3.9)-(3.10).

In our former work [8] we have determined $f\left(q^{2}\right)$ in the framework of an explicitly covariant model calculation, which we have emphasized not to be realistic. The difference between that result and Eqs.(3.9)-(3.10) for $f\left(q^{2}\right)$ is given by the contribution due to the $B$-functions in Eq.(3.9); the latter vanishes exactly if the asymmetric vertex functions of the covariant model of Ref. [8] are used. Therefore, both results are consistent.

In the standard light-front formalism only the amplitude $\hat{G}_{h^{\prime \prime}}^{+}$is used with the additional requirement that all quarks are on their respective mass shells. The corresponding expression for $f\left(q^{2}\right)$ has been derived long ago in Ref. [19] by means of a different technique and is given again in Appendix $\mathrm{C}$, together with the expression that is obtained in the CDKM approach of Ref. [2].

\subsection{The vector decay constant $f_{V}$}

The vector decay constant $f_{V}$ is defined by the matrix element of the vector current

$$
g_{h}^{\mu}=\left\langle 0\left|V^{\mu}\right| P, h\right\rangle=\varepsilon_{h}^{\mu} \sqrt{2} f_{V} .
$$

The matrix element can be represented in 1-loop order by a light-front momentum integral, which we shall denote as $\hat{g}_{h}^{\mu}$ :

$$
\hat{g}_{h}^{\mu}=\frac{N_{c}}{16 \pi^{3}} \int_{0}^{1} d x \int d^{2} p_{\perp}^{\prime} \frac{h_{0}^{\prime}}{(1-x) \hat{N}_{1}^{\prime}} s^{\mu},
$$

where

$$
s_{\mu}=\varepsilon_{h}^{\nu} \operatorname{tr}\left\{\gamma_{\mu}\left(\not p_{1}^{\prime}+m_{1}^{\prime}\right)\left[\gamma_{\nu}-\frac{1}{D^{\prime}}\left(p_{1}^{\prime}-p_{2}\right)_{\nu}\right]\left(-\not p_{2}+m_{2}\right)\right\}
$$

and we have used the vertex operator for ${ }^{3} S_{1}$-state mesons, Eq.(2.13). An explicit representation of the trace can be found in Appendix C.

The decomposition of the integral $\hat{g}_{h}^{\mu}$, Eq.(3.12), into 4-vectors depends on the light-front in the following way

$$
\hat{g}_{h}^{\mu}=\sqrt{2}\left\{\varepsilon_{h}^{\mu} f_{V}+\frac{1}{(\omega \cdot P)^{2}} \varepsilon_{h} \cdot \omega \omega^{\mu} r_{V}\right\},
$$


where the term proportional to $r_{V}$ is spurious. We have used an identity similar to Eq. (2.20)

$$
\begin{aligned}
\frac{1}{\omega \cdot P} P^{\mu} \varepsilon_{h} \cdot \omega= & \varepsilon_{h}^{\mu}+\omega^{\mu} P^{2} \frac{\varepsilon_{h} \cdot \omega}{(\omega \cdot P)^{2}} \\
& +\frac{1}{(\omega \cdot P)^{2}} \varepsilon^{\mu \alpha \beta \nu} P_{\alpha} \omega_{\beta} \varepsilon_{\nu \tau \sigma \gamma} \varepsilon_{h}^{\tau} P^{\sigma} \omega^{\gamma}
\end{aligned}
$$

in order to remove the term proportional to $P_{\mu}$, which violates current conservation, and absorb its contribution into the decay constant $f_{V}$. Again we neglect the $\varepsilon$ tensor term. The result for $f_{V}$ is

$$
\begin{array}{r}
f_{V}=\frac{N_{c}}{8 \pi^{3}} \int_{0}^{1} d x \int d^{2} p_{\perp}^{\prime} \frac{\sqrt{2} h_{0}^{\prime}}{(1-x) \hat{N}_{1}^{\prime}}\left\{x M_{0}^{\prime 2}-m_{1}^{\prime}\left(m_{1}^{\prime}-m_{2}\right)-p_{\perp}^{\prime 2}+2 B_{1}^{(2)}\right. \\
\left.+\frac{1}{D^{\prime}}\left[\left(m_{1}^{\prime}+m_{2}\right)\left(p_{\perp}^{\prime 2}-2 B_{1}^{(2)}\right)+m_{1}^{\prime} C_{1}^{(1)}\right]\right\} .
\end{array}
$$

The standard procedure uses the plus component of Eq.(3.12) for the longitutinal decay mode to evaluate the vector decay constant and reproduces the result (3.16). In Eq.(3.16) we have used the same symbols for $B_{1}^{(2)}$ and $C_{1}^{(1)}$ as in Appendix A, though the limit $q^{2}=q \cdot P=0$ must be taken.

The equation for $f_{V}$ contains a $C$-function and we use the consistency relation (2.39) in order to determine the value of $C_{1}^{(1)}$ in Eq.(3.16):

$$
C_{1}^{(1)} \doteq 0
$$

The unique result for $f_{V}$ is given by Eqs.(3.16)-(3.17).

Eqs.(3.16)-(3.17) and the result for $f_{V}$ found in Ref. [8] differ again by the contribution due to the $B$-functions in Eq.(3.16), which vanishes in the covariant model.

The standard light-front expression for the vector decay constant can be derived from the amplitude $\hat{g}_{0}^{+}$for on-shell quarks [20], and is given in Appendix C together with the formula obtained in the CDKM scheme of Ref. [2].

\section{Applications}

In Refs. [17, 19, 20] we have used the standard light-front formalism to investigate electroweak properties of light and heavy mesons, and found good agreement with the experimental data. Especially the predictions obtained in Refs. [20, 21, 22] for light pseudoscalar and vector mesons, for which a large amount of precise information is available, are remarkably good. The approach of Refs. [20, 21] is based on a Gaussian model wave function, which is parametrized in terms of adjustable constants, while [22] uses a Gaussian wave function as a trial function of the variational principle for a QCD-motivated Hamiltonian. The main difference in 
the best fit parameters used in $[20,21,22]$ was the constituent quark masses, i.e., $m_{u}=m_{d}=m=250 \mathrm{MeV}, m_{s}=370 \mathrm{MeV}$ in $[20,21]$, and $m=250 \mathrm{MeV}, m_{s}=480$ $\mathrm{MeV}$ in [22] for a confining harmonic oscillator potential.

We expect that the refinements proposed in the present work will lead to only minor modifications of this general picture. Therefore, we are mainly interested in a comparison of certain quantitative predictions of our approach with those of the covariant CDKM scheme and the standard light-front formalism. For this purpose we shall use the constituent masses $m=250 \mathrm{MeV}, m_{s}=370 \mathrm{MeV}$, however, it is interesting to calculate also the predictions of our formalism for the large s-quark mass $m_{s}=480 \mathrm{MeV}$. Once the values of the constituent masses are given, the corresponding values of the wave function parameters $\beta$ can be fixed by using the central values of the measured decay constants [23] $f_{\pi}=92.4 \pm 0.2 \mathrm{MeV}, f_{\rho} / M_{\rho}=$ $150 \pm 3.6 \mathrm{MeV}, f_{K}=113.4 \pm 1.1 \mathrm{MeV}$, and $f_{K^{*}} / M_{K^{*}}=153 \pm 3 \mathrm{MeV}$. The value for $f_{\rho} / M_{\rho}$ is an average of the values for $\rho^{ \pm}\left(f_{\rho^{ \pm}} / M_{\rho^{ \pm}}=147.1 \pm 0.88 \mathrm{MeV}\right.$ from $\operatorname{Br}(\tau \rightarrow \rho \nu)=0.250 \pm 0.003)$ and $\rho^{0}\left(f_{\rho^{0}} / M_{\rho^{0}}=152.7 \pm 3.5 \mathrm{MeV}\right.$ from $e^{+} e^{-}$ annihilation). The value for $f_{K^{*}} / M_{K^{*}}$ can be derived from the branching ratio $\operatorname{Br}\left(\tau \rightarrow K^{*} \nu\right)=0.0129 \pm 0.0005$. An experimental value for the decay constant $f_{D}$ of the charm meson has been found but with very large errors [23]. For the purpose of our calculation we shall use the values $\sqrt{2} f_{D}=203 \mathrm{MeV}$, and $\sqrt{2} f_{D^{*}} / M_{D^{*}}=$ $238 \mathrm{MeV}$. The value for $\sqrt{2} f_{D}$ [in $\left.\mathrm{MeV}\right]$ is consistent with the average lattice data obtained in quenched [203(14)] and unquenched [226(15)] approximations from the recent review [24], and the QCD sum rule predictions [203(23)], [195(20)] from Refs. $[25,26]$, respectively. The value for $\sqrt{2} f_{D^{*}} / M_{D^{*}}$ has been determined with the central value of the ratio $f_{D^{*}} /\left(f_{D} M_{D^{*}}\right)=1.17 \pm 0.06$, which can be extracted from the results of lattice calculations presented in $[27,28]$. We have listed our values for masses and wave function parameters in Table I.

\begin{tabular}{|l|l|l|l|l|l|}
\hline$(q \bar{Q})$ meson & $m_{Q}[\mathrm{GeV}]$ & $\beta_{P}[\mathrm{GeV}]$ & $\beta_{V}[\mathrm{GeV}]$ & $\beta_{V}^{S L F}[\mathrm{GeV}]$ & $\beta_{V}^{C D K M}[\mathrm{GeV}]$ \\
\hline$\pi, \rho$ & 0.25 & 0.3194 & 0.280 & 0.316 & 0.262 \\
\hline$K, K^{*}$ & 0.37 & 0.3951 & 0.296 & 0.327 & 0.280 \\
& 0.48 & 0.3629 & 0.294 & & \\
\hline$D, D^{*}$ & 1.50 & 0.4987 & 0.455 & 0.478 & 0.434 \\
\hline
\end{tabular}

Table 1: Quark masses $m_{Q}$ and wave function parameters $\beta_{P}$ and $\beta_{V}$ for $(q, \bar{Q})$ pseudoscalar and vector mesons, which we use in this work. The wave function parameters to be used in the SLF and CDKM schemes have been denoted by $\beta_{V}^{S L F}$ and $\beta_{V}^{C D K M}$, respectively. The light quark mass is $m_{q}=m_{u, d}=m=0.25 \mathrm{GeV}$.

\subsection{Electromagnetic form factors of the $\rho$ meson at $q^{2}=0$.}

The transition form factors of a vector meson have been defined in Eq.(2.1). While the determination of the light-front integrals for the form factors $F_{i}\left(q^{2}\right)(i=$ 
$1,2,3)$ is straightforward we shall calculate them only at $q^{2}=0$ for $m_{1}^{\prime}=m_{1}^{\prime \prime}=$ $m_{2}=m$ and $M^{\prime}=M^{\prime \prime}=M$.

For the evaluation of the $p_{\perp}^{\prime}$ integrals it is of advantage to change the momentum variable to $p_{\perp}=p_{\perp}^{\prime}-\frac{1}{2}(1-x) q_{\perp}$ and eliminate terms linear in $p_{\perp}$ by symmetric integration.

The form factors at $q^{2}=0$ are

$$
\begin{aligned}
F_{1}(0)= & \frac{N_{c}}{16 \pi^{3}} \int_{0}^{1} d x \int d^{2} p_{\perp} \frac{\left(h_{0}\right)^{2}}{(1-x)\left(\hat{N}_{1}\right)^{2}}\left\{2 x M_{0}^{2}\right. \\
& \left.+8 x A_{1}^{(2)}\left(1-\frac{4 m}{D}+\frac{4 m^{2}-M_{0}^{2}}{D^{2}}\right)\right\}
\end{aligned}
$$

whith

$$
M_{0}^{2}=\frac{p_{\perp}^{2}+m^{2}}{x(1-x)}, \hat{N}_{1}=x\left(M^{2}-M_{0}^{2}\right),
$$

where $h_{0}$ is given in terms of $M_{0}$ by Eq.(2.14) and $D=M_{0}+2 m$. Note that

$$
1-\frac{4 m}{D}+\frac{4 m^{2}-M_{0}^{2}}{D^{2}}=0
$$

for $D=M_{0}+2 m$. For the purpose of comparing our method with other approaches we shall use also a different expression for $D$.

Introducing the longitutinal momentum $p_{3}$ and changing variables from $\left(x, p_{\perp}\right)$ to $\left(p_{3}, p_{\perp}\right)$ with $p^{2}=p_{\perp}^{2}+p_{3}^{2}, M_{0}^{2}=4 E^{2}=4\left(m^{2}+p^{2}\right)$ and $x=\left(E+p_{3}\right) / M_{0}$ one can rewrite Eq.(4.1) for $D=M_{0}+2 m$ as

$$
F_{1}(0)=\frac{N_{c}}{(2 \pi)^{3}} \int d^{3} p\left|\phi\left(M_{0}^{2}\right)\right|^{2}=1 .
$$

This is the normalization condition for the wave function $\phi\left(M_{0}^{2}\right)$.

The remaining form factors are

$$
\begin{aligned}
F_{2}(0)= & \frac{N_{c}}{16 \pi^{3}} \int_{0}^{1} d x \int d^{2} p_{\perp} \frac{\left(h_{0}\right)^{2}}{(1-x)\left(\hat{N}_{1}\right)^{2}}\left\{-4 x M_{0}^{2}+2 p_{\perp}^{2}+2 x(1-x)\left(M^{2}-M_{0}^{2}\right)\right. \\
& -8\left[(1-x) B_{1}^{(2)}+A_{1}^{(2)}\right]\left(1-\frac{4 m}{D}+\frac{4 m^{2}-M_{0}^{2}}{D^{2}}\right) \\
& -\frac{4 m}{D}\left[x(1-x)\left(M^{2}-M_{0}^{2}\right)+p_{\perp}^{2}\right]-\left(\frac{d}{d M_{0}} \frac{1}{D}\right) \frac{2 m}{M_{0}}\left(M^{2}-M_{0}^{2}\right) p_{\perp}^{2} \\
& +\frac{4}{D^{2}}\left[2 x M^{2} p_{\perp}^{2}+x\left(M^{2}-M_{0}^{2}\right)\left((1-x) M^{2}-x M_{0}^{2}\right)\right. \\
& \left.\left.-x\left((1-x) M^{2}-x M_{0}^{2}\right)^{2}\right]\right\}, \\
F_{3}(0)= & 2 M^{2} \frac{N_{c}}{16 \pi^{3}} \int_{0}^{1} d x \int d^{2} p_{\perp} \frac{\left(h_{0}\right)^{2}}{(1-x)\left(\hat{N}_{1}\right)^{2}}\left\{\frac{4(1-x) m}{D}-\frac{2 m p_{\perp}^{2}}{x M_{0} D^{2}}\right. \\
& \left.+\frac{p_{\perp}^{4}}{x M_{0}^{2} D^{2}}\right\},
\end{aligned}
$$


where Eq.(4.4) for $F_{3}(0)$ is valid only for $D=M_{0}+2 m$. The form factors $F_{1}(0)$ and $F_{3}(0)$ are free of $C$-functions and are predicted unambiguously. Only $F_{2}(0)$ depends on $C_{1}^{(1)}$ and $Y$, and we have used the consistency relations $C_{1}^{(1)} \doteq 0$ and $Y \doteq 0$, Eqs.(2.38), (2.41), (2.42).

The specific formulas in the SLF and CDKM schemes for $D=M_{0}+2 m$ differ from Eq.(4.3). In the standard light-front formalism $F_{2}(0)$ can be extracted uniquely from the amplitudes $\hat{G}_{h^{\prime \prime} h^{\prime}}^{+}$, since the angular condition at zero-momentum transfer is fulfilled for the vector vertex operator (2.13) (e.g. Ref.[29]). This result can be derived also from Eqs.(2.24)-(2.29) for on-shell quarks and we find

$$
\Delta^{S L F}(0)=\frac{1}{M} H_{1}^{S L F}(0)=0 .
$$

For the determination of $F_{2}^{S L F}(0)$ we can apply the same method that we have used for the evaluation of $f^{S L F}(0)$. The result is

$$
F_{2}^{S L F}(0)=\frac{N_{c}}{16 \pi^{3}} \int_{0}^{1} d x \int d^{2} p_{\perp} \frac{\left(h_{0}\right)^{2}}{(1-x)\left(\hat{N}_{1}\right)^{2}}\left\{-4 x M_{0}^{2}-\frac{4 m}{D}(1-2 x) M_{0} M\right\} .
$$

In spite of Eq.(4.5), $F_{2}^{S L F}(0)$ contains spurious contributions related to unresolved zero mode effects.

If one uses the helicity amplitude $\hat{G}_{++}^{\perp}$ for on-shell quarks, i.e., again avoiding the longitutinal modes, or the representation of the traces (2.18) given in Appendix $\mathrm{B}$, omitting all $\omega$-dependent terms, one finds the same expression as in the CDKM scheme of Refs.[2, 18]:

$$
\begin{aligned}
F_{2}^{C D K M}(0) & =\frac{N_{c}}{16 \pi^{3}} \int_{0}^{1} d x \int d^{2} p_{\perp} \frac{\left(h_{0}\right)^{2}}{(1-x) x\left(\hat{N}_{1}\right)^{2}}\left\{2 p_{\perp}^{2}-2 x M_{0}^{2}-\frac{4 m}{D} p_{\perp}^{2}\right\} \\
& =\frac{N_{c}}{16 \pi^{3}} \int_{0}^{1} d x \int d^{2} p_{\perp} \frac{\left(h_{0}\right)^{2}}{(1-x)\left(\hat{N}_{1}\right)^{2}}\left\{-4 x M_{0}^{2}-\frac{4 m}{D}(1-2 x) M_{0}^{2}\right\} .
\end{aligned}
$$

The form factors $F_{i}$ at $q^{2}=0$ are related to the static multipole moments of the charged vector meson by

$$
F_{1}(0)=1, F_{2}(0)=-\mu, F_{1}(0)+F_{2}(0)+F_{3}(0)=Q,
$$

where $\mu$ is the magnetic moment (in units of $e / 2 M$ ) and $Q$ the electric quadrupole moment (in units of $e / M^{2}$ ). As a reference we shall use the natural magnetic and quadrupole moment of a composite spin-1 system which have been derived in [14] in the limit that the system becomes pointlike:

$$
\mu=2, Q=-1 \quad \text { (point limit) . }
$$

Non-zero values for $\mu-2$ and $F_{3}(0)$ define the anomalous moments, dynamical contributions which are strictly due to internal structure [14]. 
The magnetic and quadrupole moments of the rho meson, denoted as $\mu_{\rho}$ and $Q_{\rho}$, respectively, can be calculated in the different schemes with Eqs.(4.3)-(4.7). With $M_{\rho}=769 \mathrm{MeV}$ and the quark model parameters $\left(m, \beta_{\rho}\right)$ given in Table I, we find the results collected in Table II.

\begin{tabular}{|l|l|l|l|}
\hline & this work & SLF & CDKM \\
\hline$\mu_{\rho}=-F_{2}(0)$ & 1.83 & 2.23 & 2.23 \\
\hline$F_{3}(0)$ & 1.16 & 1.04 & 1.23 \\
\hline$Q_{\rho}=F_{1}(0)+F_{2}(0)+F_{3}(0)$ & 0.33 & -0.19 & -0.005 \\
\hline
\end{tabular}

Table 2: Magnetic and quadrupole moments of the charged rho meson calculated in this work are compared with the results obtained in the SLF and CDKM schemes.

The anomalous quadrupole moment consists of a large contribution due to $F_{3}(0)$, which is predicted unambiguously by the standard light-front formalism, and a small contribution due to the anomalous magnetic moment $\mu_{\rho}-2$, which depends upon the underlying scheme.

The SLF and CDKM values for $\mu_{\rho}$ in Table II are consistent with the results ( $\mu_{\rho}=2.26$ and $\mu_{\rho}=2.35$ ) obtained in Refs. [29] and [18], respectively, using a $q \bar{q}$ wave function generated by a quark potential and a quark mass $m=0.22 \mathrm{GeV}$.

The different values for the magnetic moment of the rho are particularly remarkable, and it is interesting to compare our results with previous estimates for $\mu_{\rho}$. The nonrelativistic $\mathrm{SU}(6)$ model, which neglects all interaction effects, predicts

$$
\mu_{\rho}=2 \quad(\mathrm{SU}(6) \text { value }),
$$

in accordance with (4.8). This relation is obtained also in the formalism of hard pions with current algebra [30], and in the framework of the Lagrangian formulation of the vector meson dominance hypothesis [31] if strong interaction is neglected [32]. Inclusion of quark confinement leads to deviations from Eq.(4.9), i.e. to an anomalous magnetic moment. In the SLF and CDKM schemes of the light-front formalism one finds $\mu_{\rho}>2$, in qualitative agreement with the results derived in models based upon the QCD Dyson-Schwinger equation [33, 34].

In contrast to this picture, our light-front approach, which includes the effect of zero modes, predicts $\mu_{\rho}<2$, which qualitatively agrees with estimates derived from an analysis of early SLAC and DESY data for charged $\rho$ photoproduction [35], and the result of a recent calculation in the framework of QCD sum rules [32] $\left(\alpha_{s}\right.$-corrections are, however, neglected). We mention also an oldfashioned phenomenological approach to estimate $\mu_{\rho}$ : The nonrelativistic SU(6) model not only predicts the magnetic moment of the rho, we write the result as $\mu_{\rho}=\mu$, but also the rate of the radiative decay of vector mesons, for example $\omega \rightarrow \pi+\gamma$. This rate can be expressed in terms of $\mu$ as (e.g. Ref. [36])

$$
\Gamma(\omega \rightarrow \pi \gamma)=\frac{1}{3 \pi}\left(\mu \frac{e}{2 M_{\rho}}\right)^{2}\left(\frac{M_{\omega}^{2}-M_{\pi}^{2}}{2 M_{\omega}}\right)^{3} .
$$


We have omitted the overlap integral of the product of pion and $\omega(\rho)$ wave functions, introduced by Isgur in Ref. [37], since it is very close to 1 (if the parameters of Table I are used). With $\Gamma(\omega \rightarrow \pi \gamma)=0.72 \pm 0.04 \mathrm{MeV}$, Ref.[23], and treating $\mu$ as a free parameter, one finds $\mu_{\rho}=1.79 \pm 0.05$, close to our value given in Table II.

In order to gain some insight into the physical mechanism that determines the size of the magnetic moment of the rho, we shall use the toy model, which we analyzed in Ref. [8], as a guide. Based upon a special class of meson vertex functions we have shown in [8] that there exists an exact correspondence between the explicitly covariant 4-dimensional and the light-front calculations in 1-loop order. While the vertex functions used in the approach [8] are not symmetric in the 4-momenta of the constituent quarks, and can hardly be considered a realistic approximation of the $q \bar{q}$ bound state, we expect that the general features of a covariant formalism are reproduced correctly.

If we use the vector vertex operator (2.13), the toy model helicity amplitude is given by Eq.(2.17) with the asymmetric vertex function

$$
h_{0}^{\prime}=\frac{Z^{\prime}}{\hat{N}_{1}^{\prime}-\Lambda^{2}+m^{2}} \quad \text { (monopole form of the toy model), }
$$

where $\Lambda$ is a cut-off parameter. A similar equation holds for $h_{0}^{\prime \prime}$, and we choose $D^{\prime}=D^{\prime \prime}=D=M_{\rho}+2 m$. The normalization condition $F_{1}(0)=1$, where $F_{1}(0)$ is given by Eq.(4.1), fixes the constant $Z^{\prime}$, and the toy model value, denoted by $\mu_{\rho}^{\text {toy }}$, can be computed from Eq.(4.3) for $F_{2}(0)$. Note that the consistency conditions for C-functions that have been used for the derivation of the formula (4.3) are automatically fulfilled in the toy model. For the numerical calculation we use the same parameters as in Ref. [15], $m=0.43 \mathrm{GeV}$ and $\Lambda=1.8 \mathrm{GeV}$, and include first only the $\gamma$-term of the vector vertex operator, and second the full vertex structure of Eq.(2.13). We give the results for $\mu_{\rho}^{\text {toy }}$ together with those obtained in this work:

$$
\left(\mu_{\rho}^{\text {toy }}, \mu_{\rho}^{\text {thiswork }}\right)= \begin{cases}(2.12,2.17) & \text { (with only the first term of the vertex }(2.13)) \\ (1.79,1.83) & \text { (with both terms of the vertex }(2.13)) .\end{cases}
$$

Obviously, the predictions of both approaches are in qualitative agreement. This calculation indicates that the detailed structure of the vector vertex influences the size of $\mu_{\rho}$, and that the second term of the structure (2.13) is responsible for the result $\mu_{\rho}<2$. We emphasize that the toy model is explicitly covariant and automatically includes zero mode contributions, while the method which we have presented in this work accounts for the effect of zero modes in terms of specific consistency relations. The SLF and CDKM schemes also use the full structure of the vector vertex (2.13), but ignore zero mode effects, which might be the reason for the result $\mu_{\rho}>2$.

\subsection{The transition $V \rightarrow P+\pi^{0}$.}

In Ref. [17] we have investigated the pionic decays $\rho^{+} \rightarrow \pi^{+} \pi^{0}, K^{*+} \rightarrow(K \pi)^{+}$ and $D^{*+} \rightarrow(D \pi)^{+}$, and have calculated the coupling constant $g_{V P \pi^{0}}$ by means of a 
soft pion theorem due to Das, Mathur and Okubo [38]

$$
4\left|g_{V P \pi^{0}}\right| f_{\pi}=\left|f(0)-\left(M_{V}^{2}-M_{P}^{2}\right) a_{+}(0)\right|
$$

where $a_{+}(0)$ and $f(0)$ are given by Eqs.(3.8) and (3.9), and the SLF and CDKM expressions for $f(0)$ by Eqs.(C.3)-(C.4). The pion decay constant is $f_{\pi}=92.4 \mathrm{MeV}$. (Note, that if this relation refers to $g_{\rho^{+} \pi^{+} \pi^{0}}$ the factor 4 in eq.(4.10) must be replaced by the factor 2.) The rate for the decay $V \rightarrow P+\pi$ is given by

$$
\begin{gathered}
\Gamma=g_{V P \pi}^{2} p^{3} /\left(6 \pi M_{V}^{2}\right) \\
p^{2}=\left[M_{V}^{2}-\left(M_{P}+M_{\pi}\right)^{2}\right]\left[M_{V}^{2}-\left(M_{P}-M_{\pi}\right)^{2}\right] /\left(4 M_{V}^{2}\right) .
\end{gathered}
$$

It might be interesting to compare the coupling constants predicted by our method, and by the SLF and CDKM schemes, with the data, and we have collected the corresponding numerical results in Table III. The coupling constants for different charge states are related by

$$
\begin{aligned}
g_{\rho \pi \pi} & \equiv g_{\rho^{+} \pi^{+} \pi^{0}} \\
g_{K^{*} K \pi} & \equiv \sqrt{3} g_{K^{*+} K^{+} \pi^{0}}=\sqrt{3} g_{K^{*+} K^{0} \pi^{+}} / \sqrt{2} \\
g_{D^{*} D \pi} & \equiv g_{D^{*+} D^{+} \pi^{0}}=g_{D^{*+} D^{0} \pi^{+}} / \sqrt{2}
\end{aligned}
$$

The $D^{*} D \pi$ coupling constant is usually expressed in terms of a universal strong coupling constant $g$, for the decay of a heavy vector meson into a heavy pseudoscalar meson and a pion, with

$$
g=2 g_{D^{*} D \pi} f_{\pi} / M_{D^{*}}
$$

The decay rate $\Gamma_{t o t}\left(D^{*+}\right)$ is given by

$$
\Gamma_{t o t}\left(D^{*+}\right)=\Gamma\left(D^{*+} \rightarrow(D \pi)^{+}\right)+\Gamma\left(D^{*+} \rightarrow D^{+} \gamma\right) .
$$

The pionic decay rate $\Gamma\left(D^{*+} \rightarrow(D \pi)^{+}\right)$can be calculated with Eq.(4.11). The (small) radiative decay rate $\Gamma\left(D^{*+} \rightarrow D^{+} \gamma\right)$ depends on the form factor $V(0)$, which is free of C-functions, and for its evaluation we have used the results of Ref. [17].

A discussion of $g$ in the SLF scheme can be found in Ref. [39]. For no Melosh rotation and $\beta_{D}=\beta_{D^{*}}$ one would get $g=1$ as in the nonrelativistic quark model [40]. In the framework of the light-front formalism the Melosh rotation generates the relativistic spinor structure of the vertex operators (2.13) and (3.4) and generally one obtains $g<1$. The precise value of $g$ depends upon the relative size of the constituent masses. For example, for a small (yet unrealistic) constituent mass $m_{u / d}=100 \mathrm{MeV}$ one finds the unlikely value $g \simeq 0.3$ (this work and SLF). If the constituent mass approaches zero $g$ gets very small. For the set of parameters given in Table I the agreement of the predictions of our approach and of the SLF scheme with the data is satisfactory and within the range expected from the effect of an underlying approximate chiral symmetry. The predictions based upon the 


\begin{tabular}{|l|l|l|l|l|}
\hline & this work & SLF & CDKM & experiment \\
\hline$g_{\rho \pi \pi}$ & 6.10 & 5.62 & 10.0 & $6.06 \pm 0.01[23]$ \\
\hline$g_{K^{*} K \pi}$ & 5.43 & 5.20 & 9.41 & $5.57 \pm 0.03[23]$ \\
& 5.85 & & & \\
\hline$g_{D^{*} D \pi}$ & 6.24 & 6.22 & 11.16 & $6.3 \pm 0.1 \pm 0.7[43]$ \\
\hline$g$ & 0.57 & 0.57 & 1.03 & $0.59 \pm 0.01 \pm 0.07[43]$ \\
\hline$\Gamma_{\text {tot }}\left(D^{*+}\right)[\mathrm{keV}]$ & 93.0 & 92.4 & 295.7 & $96 \pm 4 \pm 22[43]$ \\
\hline
\end{tabular}

Table 3: Coupling constants for $V \rightarrow P \pi$ decays, and the total rate for the decay of the charged $D^{*}$ meson. The parameters of Table I have been used.

CDKM scheme are surprisingly large, which seems to indicate that the prescriptions used for the calculation of $f^{C D K M}\left(q^{2}\right)$ are probably too restrictive, i.e. we suspect that either the on-mass-shell prescription or the prescription that all $\omega$-dependent contributions should be omitted, is responsible for these results. In this context it may be interesting to note that within the CDKM scheme it has been found for the deuteron electrodisintegration [41] that instantaneous contributions can be large, and for nucleon form factors [42] that $\omega$-dependent structures can give contributions to physical form factors.

Of particular importance is the recent measurement of the total decay rate of the charged $D^{*}$ meson by the CLEO collaboration [43], since the coupling constant $g_{D^{*} D \pi}$ is of considerable theoretical interest and has been studied extensively in past work (an overview and a list of references can be found in $[44,45]$ ). The range of published values for $g_{D^{*} D \pi}$ is quite large; we only mention the recent lattice QCD result $g_{D^{*} D \pi}=6.6 \pm 0.8_{-0.7}^{+0.4}[46]$, which is consistent with the data (and with our value given in Table III), and the results based upon various QCD sum rule techniques [47] (for example Ref. [48] predicts $g_{D^{*} D \pi}=3.7 \pm 1.1$, i.e., $g=0.34$ close to the "unlikely" light-front value), which are much smaller than the data. However, in a recent reanalysis of the $D^{*} D \pi$ coupling in the framework of the light-cone QCD sum rule [49] it was pointed out how agreement with the data could be attained (another attempt has been published in [50]).

It would be interesting to explore the connection between the light-front formalism and the light-cone QCD sum rule approach.

\subsection{Determination of current quark masses.}

The masses of the quarks in the light-front formalism are the constituent masses. Even in this framework it is possible to obtain an estimate of the current quark masses from the Ward identity for the axial current. It can be derived starting from the matrix element of the axial current between the vacuum and a $q \bar{q}$ meson

$$
\left\langle 0\left|\bar{q}^{\prime \prime} \gamma_{\mu} \gamma_{5} q^{\prime}\right| P\right\rangle=i P_{\mu} \sqrt{2} f_{P},
$$


and is given by [51]

$$
-\left(\hat{m}_{q^{\prime}}+\hat{m}_{q^{\prime \prime}}\right)\left\langle 0\left|\bar{q}^{\prime \prime} \gamma_{5} q^{\prime}\right| P\right\rangle=i \sqrt{2} f_{P} M_{P}^{2}
$$

This relation involves the current quark masses $\hat{m}_{q}$ and is exact, except for electroweak corrections.

In analogy to Eq.(4.12) we define the coupling constant $g_{P}$ of the pseudoscalar density as

$$
\left\langle 0\left|\bar{q}^{\prime \prime} \gamma_{5} q^{\prime}\right| P\right\rangle=-i \sqrt{2} g_{P}
$$

and Eq.(4.13) can be rewritten as an equation for the quark masses

$$
\hat{m}_{q^{\prime}}+\hat{m}_{q^{\prime \prime}}=M_{P}^{2} f_{P} / g_{P}
$$

It is not possible to measure the coupling constant $g_{P}$, but the formalism presented in this work permits a reliable determination of the matrix element (4.14) in terms of constituent masses $m_{q}$ and wave function parameters $\beta$. Since the free parameters $\left(m_{q}, \beta\right)$ are fixed by comparison with the data, Eq.(4.15) provides an interesting phenomenological link between the current quark masses $\hat{m}_{q}$ and experiment.

The equation for the pseudoscalar decay constant $f_{P}$ can be found e.g. in [8], and the matrix element (4.14) is given in the 1-loop approximation as a light-front integral; the corresponding expression for $g_{P}$ is

$$
g_{P}=\frac{N_{c}}{16 \pi^{3}} \int_{0}^{1} d x \int d^{2} p_{\perp}^{\prime} \frac{\sqrt{2} h_{0}^{\prime}}{2(1-x) \hat{N}_{1}^{\prime}} s
$$

where

$$
\begin{aligned}
s & =\operatorname{tr}\left\{\gamma_{5}\left(\not p_{1}^{\prime}+m_{1}^{\prime}\right) \gamma_{5}\left(-\not p_{2}+m_{2}\right)\right\} \\
& =2\left(M_{P}^{2}-N_{1}^{\prime}-N_{2}-\left(m_{1}^{\prime}-m_{2}\right)^{2}\right) .
\end{aligned}
$$

With Eqs.(A.3)-(A.4) and(A.13) we find for the trace

$$
s=2\left(2 x M_{0}^{\prime 2}-2 m_{1}^{\prime}\left(m_{1}^{\prime}-m_{2}\right)+C_{1}^{(1)}\right) .
$$

Obviously $g_{P}$ is associated with zero modes. The final result for $g_{P}$ is

$$
g_{P}=\frac{N_{c}}{8 \pi^{3}} \int_{0}^{1} d x \int d^{2} p_{\perp}^{\prime} \frac{\sqrt{2} h_{0}^{\prime}}{2(1-x) \hat{N}_{1}^{\prime}}\left(x M_{0}^{\prime 2}-m_{1}^{\prime}\left(m_{1}^{\prime}-m_{2}\right)\right),
$$

where we have used the consistency relation $(2.39)$ in order to eliminate $C_{1}^{(1)}$. Note that the on-mass-shell prescription (all quarks are on their mass shells) gives the same result for $g_{P}$; the latter has been derived in this manner in Ref. [52].

If the integral (4.17) is evaluated with the quark model parameters of Table I, we find, using Eq.(4.15) with the average mass squared $M_{P}^{2}=\left(M_{P^{0}}^{2}+M_{P^{+}}^{2}\right) / 2$ :

$$
\hat{m}=\frac{1}{2}\left(\hat{m}_{u}+\hat{m}_{d}\right)=4.39 \mathrm{MeV} \quad(\text { for } \mathrm{m}=250 \mathrm{MeV})
$$




$$
\hat{m}_{s}+\hat{m}= \begin{cases}91.96 \mathrm{MeV} & \left(\text { for } \mathrm{m}_{\mathrm{s}}=370 \mathrm{MeV}\right) \\ 106.36 \mathrm{MeV} & \left(\text { for } \mathrm{m}_{\mathrm{s}}=480 \mathrm{MeV}\right) .\end{cases}
$$

Of particular interest are the estimates for the ratio $\hat{m}_{s} / \hat{m}$

$$
\hat{m}_{s} / \hat{m}= \begin{cases}19.97 & \left(\text { for } \mathrm{m}_{\mathrm{s}}=370 \mathrm{MeV}\right) \\ 23.25 & \left(\text { for } \mathrm{m}_{\mathrm{s}}=480 \mathrm{MeV}\right)\end{cases}
$$

which can be compared with the result obtained in chiral perturbation theory at the next-to-leading order [53]

$$
\hat{m}_{s} / \hat{m}=24.4 \pm 1.5 .
$$

Evidently only the large value of the constituent mass of the s-quark is consistent with (4.18).

\section{Concluding remarks}

We have proposed in this work a method to account for the effect of zero modes that are naturally associated with the light-front integrals that represent the matrix elements of a one-body current. In order to identify that part of the light-front integral that is influenced by zero modes, we have decomposed it in terms of the 4-vectors $P, q$ and $\omega$, and found that the decomposition coefficients $C_{n}^{(m)}$ must be completed by (unknown) zero mode contributions. Fortunately there are only two combinations of C-functions, namely $C_{1}^{(1)}$ and $Y$, that are common to all form factors that are affected by zero modes. With plausible assumptions it is possible to derive consistency conditions for the functions $C_{1}^{(1)}$ and $Y$, which permit an unambiguous determination of the C-dependent form factors. This method provides a practical scheme to calculate the effect of zero modes, and constitutes a covariant extension of the standard light-front formalism.

The correctness of this procedure is tested by the phenomenological success of the derived form factors. In practical applications it appears that the predictions of the SLF approach and the covariant extension proposed in this work are usually very similar, i.e., the good agreement with the data found in the SLF framework is confirmed. However, there are problems like the $q^{2}$ dependence of the electromagnetic form factors of vector mesons, or the details of the magnetic moment of the rho that can be treated only by the refined formalism.

Based upon rules, similar to those established for $q \bar{q}$ mesons, it should be possible to determine also zero-mode-affected form factors of $q q q$ baryons. For example, it is

remarkable that the charge form factor of the proton is free of C-functions, but its magnetic form factor depends on C-functions in terms of expressions corresponding to $C_{1}^{(1)}$ and $Y$.

\section{Acknowledgements}

I would like to thank Daniel Wyler for helpful discussions. 


\section{A Appendix: Decomposition of tensors}

In order to treat the Lorentz structure of a hadronic matrix element the authors of Ref. [2] have developed a method to identify and separate spurious contributions and to determine the physical part of the hadronic matrix element. We have developed an essentially different technique in Ref. [8] to deal with this problem. It is based on the decomposition of 4 -tensors with regard to $P, q$ and $\omega$; we shall copy the respective formulas which we shall need in the present work from Ref. [8].

The integrand of a light-front integral, like Eq.(2.17), depends on the tensors $p_{1 \mu}^{\prime}, p_{1 \mu}^{\prime} p_{1 \nu}^{\prime}$ and $p_{1 \mu}^{\prime} p_{1 \nu}^{\prime} p_{1 \alpha}^{\prime}$. Let us start with the 4 -vector $p_{1 \mu}^{\prime}$ whose decomposition is inferred from symmetry considerations to be

$$
\begin{array}{r}
\int_{0}^{1} d x \int d^{2} p_{\perp}^{\prime} \frac{R^{\prime} S^{\prime \prime}}{(1-x) \hat{N}_{1}^{\prime} \hat{N}_{1}^{\prime \prime}} p_{1 \mu}^{\prime}=\int_{0}^{1} d x \int d^{2} p_{\perp}^{\prime} \frac{R^{\prime} S^{\prime \prime}}{(1-x) \hat{N}_{1}^{\prime} \hat{N}_{1}^{\prime \prime}}\left(P_{\mu} A_{1}^{(1)}+q_{\mu} A_{2}^{(1)}\right. \\
\left.+\frac{1}{\omega \cdot P} \omega_{\mu} C_{1}^{(1)}\right)
\end{array}
$$

where $R^{\prime}$ and $S^{\prime \prime}$ are functions of $M_{0}^{\prime}$ and $M_{0}^{\prime \prime}$, respectively.

An equation like (A.1) will be written in the following as a relation between integrands as

$$
p_{1 \mu}^{\prime} \doteq P_{\mu} A_{1}^{(1)}+q_{\mu} A_{2}^{(1)}+\frac{1}{\omega \cdot P} \omega_{\mu} C_{1}^{(1)} .
$$

The coefficients in (A.1) and (A.2) are given by

$$
A_{1}^{(1)}=\frac{x}{2} \quad, \quad A_{2}^{(1)}=\frac{x}{2}-\frac{p_{\perp}^{\prime} q_{\perp}}{q^{2}} \quad, \quad C_{1}^{(1)}=-N_{2}+Z_{2},
$$

where

$$
Z_{2}=-x M_{0}^{\prime 2}+m_{1}^{\prime 2}-m_{2}^{2}+(1-x) M^{\prime 2}+\left(q^{2}+q \cdot P\right) \frac{p_{\perp}^{\prime} q_{\perp}}{q^{2}} .
$$

Note that only the coefficient which is combined with $\omega_{\mu}$, namely $C_{1}^{(1)}$, depends on $p_{1}^{\prime-}$.

We shall need also the tensor decomposition

$$
\begin{aligned}
p_{1 \mu}^{\prime} p_{1 \nu}^{\prime} \doteq & g_{\mu \nu} A_{1}^{(2)}+P_{\mu} P_{\nu} A_{2}^{(2)}+\left(P_{\mu} q_{\nu}+q_{\mu} P_{\nu}\right) A_{3}^{(2)}+q_{\mu} q_{\nu} A_{4}^{(2)} \\
& +\frac{1}{\omega \cdot P}\left(P_{\mu} \omega_{\nu}+\omega_{\mu} P_{\nu}\right) B_{1}^{(2)}+\frac{1}{\omega \cdot P}\left(q_{\mu} \omega_{\nu}+\omega_{\mu} q_{\nu}\right) C_{1}^{(2)} \\
& +\frac{1}{(\omega \cdot P)^{2}} \omega_{\mu} \omega_{\nu} C_{2}^{(2)}
\end{aligned}
$$

where

$$
A_{1}^{(2)}=-p_{\perp}^{2}-\frac{\left(p_{\perp}^{\prime} q_{\perp}\right)^{2}}{q^{2}} \quad, \quad A_{2}^{(2)}=\left(A_{1}^{(1)}\right)^{2}
$$




$$
\begin{aligned}
& A_{3}^{(2)}=A_{1}^{(1)} A_{2}^{(1)}, \quad A_{4}^{(2)}=\left(A_{2}^{(1)}\right)^{2}-\frac{1}{q^{2}} A_{1}^{(2)} \\
& B_{1}^{(2)}=A_{1}^{(1)} C_{1}^{(1)}-A_{1}^{(2)}, \quad C_{1}^{(2)}=A_{2}^{(1)} C_{1}^{(1)}+\frac{q \cdot P}{q^{2}} A_{1}^{(2)} \\
& C_{2}^{(2)}=\left(C_{1}^{(1)}\right)^{2}+\left[P^{2}-\frac{(q \cdot P)^{2}}{q^{2}}\right] A_{1}^{(2)} .
\end{aligned}
$$

Since the zero mode term of $x N_{2}$ vanishes we find the following relation:

$$
x N_{2} \doteq x \hat{N}_{2}=0
$$

and there are no zero-mode contributions associated with $B_{1}^{(2)}$, which is therefore given by its value at the spectator quark pole:

$$
B_{1}^{(2)}=\frac{x}{2} Z_{2}-A_{1}^{(2)}
$$

For the practical applications considered in this work we shall need also the decomposition of the following tensor product

$$
\begin{aligned}
p_{1 \mu}^{\prime} p_{1 \nu}^{\prime} p_{1 \alpha}^{\prime} \doteq & \left(g_{\mu \nu} P_{\alpha}+g_{\mu \alpha} P_{\nu}+g_{\nu \alpha} P_{\mu}\right) A_{1}^{(3)}+\left(g_{\mu \nu} q_{\alpha}+g_{\mu \alpha} q_{\nu}+g_{\nu \alpha} q_{\mu}\right) A_{2}^{(3)} \\
& +P_{\mu} P_{\nu} P_{\alpha} A_{3}^{(3)}+\left(P_{\mu} P_{\nu} q_{\alpha}+P_{\mu} q_{\nu} P_{\alpha}+q_{\mu} P_{\nu} P_{\alpha}\right) A_{4}^{(3)} \\
& +\left(q_{\mu} q_{\nu} P_{\alpha}+q_{\mu} P_{\nu} q_{\alpha}+P_{\mu} q_{\nu} q_{\alpha}\right) A_{5}^{(3)}+q_{\mu} q_{\nu} q_{\alpha} A_{6}^{(3)} \\
& +\frac{1}{\omega \cdot P}\left(P_{\mu} P_{\nu} \omega_{\alpha}+P_{\mu} \omega_{\nu} P_{\alpha}+\omega_{\mu} P_{\nu} P_{\alpha}\right) B_{1}^{(3)} \\
& +\frac{1}{\omega \cdot P}\left[\left(P_{\mu} q_{\nu}+q_{\mu} P_{\nu}\right) \omega_{\alpha}+\left(P_{\mu} q_{\alpha}+q_{\mu} P_{\alpha}\right) \omega_{\nu}\right. \\
& \left.+\left(P_{\nu} q_{\alpha}+q_{\nu} P_{\alpha}\right) \omega_{\mu}\right] B_{2}^{(3)} \\
& +\frac{1}{\omega \cdot P}\left(g_{\mu \nu} \omega_{\alpha}+g_{\mu \alpha} \omega_{\nu}+g_{\nu \alpha} \omega_{\mu}\right) C_{1}^{(3)} \\
& +\frac{1}{\omega \cdot P}\left(q_{\mu} q_{\nu} \omega_{\alpha}+q_{\mu} \omega_{\nu} q_{\alpha}+\omega_{\mu} q_{\nu} q_{\alpha}\right) C_{2}^{(3)} \\
& +\frac{1}{(\omega \cdot P)^{2}}\left(\omega_{\mu} \omega_{\nu} P_{\alpha}+\omega_{\mu} P_{\nu} \omega_{\alpha}+P_{\mu} \omega_{\nu} \omega_{\alpha}\right) C_{3}^{(3)} \\
& +\frac{1}{(\omega \cdot P)^{2}}\left(\omega_{\mu} \omega_{\nu} q_{\alpha}+\omega_{\mu} q_{\nu} \omega_{\alpha}+q_{\mu} \omega_{\nu} \omega_{\alpha}\right) C_{4}^{(3)} \\
& +\frac{1}{(\omega \cdot P)^{3}} \omega_{\mu} \omega_{\nu} \omega_{\alpha} C_{5}^{(3)}
\end{aligned}
$$

The coefficients are given by

$$
\begin{aligned}
& A_{1}^{(3)}=A_{1}^{(1)} A_{1}^{(2)} \quad, \quad A_{2}^{(3)}=A_{2}^{(1)} A_{1}^{(2)}, \\
& A_{3}^{(3)}=A_{1}^{(1)} A_{2}^{(2)} \quad, \quad A_{4}^{(3)}=A_{2}^{(1)} A_{2}^{(2)} \text {, } \\
& A_{5}^{(3)}=A_{1}^{(1)} A_{4}^{(2)} \quad, \quad A_{6}^{(3)}=A_{2}^{(1)} A_{4}^{(2)}-\frac{2}{q^{2}} A_{2}^{(1)} A_{1}^{(2)} \text {, }
\end{aligned}
$$




$$
\begin{aligned}
& B_{1}^{(3)}=A_{1}^{(1)} B_{1}^{(2)}-A_{1}^{(1)} A_{1}^{(2)} \quad, \quad B_{2}^{(3)}=A_{1}^{(1)} C_{1}^{(2)}-A_{2}^{(1)} A_{1}^{(2)}, \\
& C_{1}^{(3)}=A_{1}^{(2)} C_{1}^{(1)} \quad, \quad C_{2}^{(3)}=A_{4}^{(2)} C_{1}^{(1)}+2 \frac{q \cdot P}{q^{2}} A_{2}^{(1)} A_{1}^{(2)}, \\
& C_{3}^{(3)}=A_{1}^{(1)}\left(C_{1}^{(1)}\right)^{2}-2 C_{1}^{(3)}+\left(P^{2}-\frac{(q \cdot P)^{2}}{q^{2}}\right) A_{1}^{(1)} A_{1}^{(2)}, \\
& C_{4}^{(3)}=A_{2}^{(1)}\left(C_{1}^{(1)}\right)^{2}+2 \frac{q \cdot P}{q^{2}} C_{1}^{(3)}+\left(P^{2}-\frac{(q \cdot P)^{2}}{q^{2}}\right) A_{2}^{(1)} A_{1}^{(2)} .
\end{aligned}
$$

We do not require the coefficient $C_{5}^{(3)}$. Again the coefficients $B_{1}^{(3)}$ and $B_{2}^{(3)}$ are not associated with zero-mode contributions and are given by their values at the spectator quark pole:

$$
\begin{aligned}
B_{1}^{(3)} & =\frac{x}{2}\left(B_{1}^{(2)}-A_{1}^{(2)}\right) \\
B_{2}^{(3)} & =\left(\frac{x}{2}-\frac{p_{\perp}^{\prime} q_{\perp}}{q^{2}}\right) B_{1}^{(2)}+\frac{x}{2} \frac{q \cdot P}{q^{2}} A_{1}^{(2)} .
\end{aligned}
$$

Useful relations can be derived from the tensor decompositions (A.5) and (A.9):

$$
N_{1}^{\prime}=p_{1}^{\prime 2}-m_{1}^{\prime 2}=x\left(M^{\prime 2}-M_{0}^{\prime 2}\right),
$$

in accordance with Eq.(2.12), and

$$
\begin{aligned}
N_{1}^{\prime} C_{1}^{(1)}= & 2 C_{3}^{(3)}+6 C_{1}^{(3)}+q^{2} C_{2}^{(3)}-m_{1}^{\prime 2} C_{1}^{(1)}+P^{2} B_{1}^{(3)}+2 q \cdot P B_{2}^{(3)} \\
N_{1}^{\prime \prime} C_{1}^{(1)}= & 2 C_{3}^{(3)}+6 C_{1}^{(3)}+q^{2} C_{2}^{(3)}-2 q^{2} C_{1}^{(2)} \\
& +\left(q^{2}-m_{1}^{\prime 2}\right) C_{1}^{(1)}+P^{2} B_{1}^{(3)}+2 q \cdot P B_{2}^{(3)}-2 q \cdot P B_{1}^{(2)}
\end{aligned}
$$

These relations can be combined with Eq.(2.26) for $Y$ to give

$$
\begin{aligned}
& \left(N_{1}^{\prime}+N_{1}^{\prime \prime}\right) C_{1}^{(1)}=2 Y+4 C_{3}^{(3)}+8 C_{1}^{(3)}+\left(q^{2}-m_{1}^{\prime 2}-m_{1}^{\prime \prime 2}\right) C_{1}^{(1)} \\
& \left(N_{1}^{\prime}-N_{1}^{\prime \prime}\right) C_{1}^{(1)}=q^{2}\left(2 C_{1}^{(2)}-C_{1}^{(1)}\right)-\left(m_{1}^{\prime 2}-m^{\prime \prime 2}\right) C_{1}^{(1)}+2 q \cdot P B_{1}^{(2)} .
\end{aligned}
$$

In the same manner we find

$$
\begin{aligned}
N_{2} C_{1}^{(1)}= & 2 C_{3}^{(3)}+6 C_{1}^{(3)}+q^{2} C_{2}^{(3)}-C_{2}^{(2)}-\left(q^{2}+q \cdot P\right) C_{1}^{(2)} \\
& +\left(M^{\prime 2}-m_{2}^{2}\right) C_{1}^{(1)}+P^{2} B_{1}^{(3)}+2 q \cdot P B_{2}^{(3)}-\left(P^{2}+q \cdot P\right) B_{1}^{(2)}, \\
N_{2} B_{1}^{(2)}= & -C_{3}^{(3)}-C_{1}^{(3)}+B_{3}^{(3)}
\end{aligned}
$$

where

$$
B_{3}^{(3)}=B_{1}^{(2)} Z_{2}+\left(P^{2}-\frac{(q \cdot P)^{2}}{q^{2}}\right) A_{1}^{(1)} A_{1}^{(2)}
$$




\section{B Appendix: The traces of Eq.(2.18)}

Using the tensor decomposition of Appendix A the traces of Eq.(2.18) can be represented for $m_{1}^{\prime}=m_{1}^{\prime \prime}=m_{2}=m$ as

$$
\begin{aligned}
& A^{\mu}=\varepsilon_{h^{\prime \prime}}^{*} \cdot \varepsilon_{h^{\prime}} P^{\mu}\left\{8 x A_{1}^{(2)}+(1-x) q^{2}+x\left(M_{0}^{\prime 2}+M_{0}^{\prime \prime 2}\right)\right\} \\
& +\left(\varepsilon_{h^{\prime \prime}}^{*} \cdot P\right)\left(\varepsilon_{h^{\prime}} \cdot P\right) P^{\mu} 8 x\left(A_{2}^{(2)}-A_{4}^{(2)}+A_{2}^{(1)}-A_{1}^{(1)}\right) \\
& +\left(\varepsilon_{h^{\prime}}^{\mu} \varepsilon_{h^{\prime \prime}}^{*} \cdot P+\varepsilon_{h^{\prime \prime}}^{* \mu} \varepsilon_{h^{\prime}} \cdot P\right)\left\{16 A_{1}^{(3)}-8 A_{1}^{(2)}-2 C_{1}^{(1)}-4 x M_{0}^{\prime 2}\right. \\
& -x(1-x)\left(M_{0}^{\prime 2}+M_{0}^{\prime \prime 2}\right)+x(1-x)\left(M^{\prime 2}+M^{\prime \prime 2}\right)+2 x\left(M_{0}^{\prime 2}-M_{0}^{\prime \prime 2}\right) A_{2}^{(1)} \\
& \left.-2 q^{2} A_{2}^{(1)}-(1-2 x) q^{2}+x\left(1-2 A_{2}^{(1)}\right) q \cdot P\right\} \\
& +\left(\varepsilon_{h^{\prime}}^{\mu} \varepsilon_{h^{\prime \prime}}^{*} \cdot P-\varepsilon_{h^{\prime \prime}}^{* \mu} \varepsilon_{h^{\prime}} \cdot P\right)\left\{16 A_{2}^{(3)}-8 A_{1}^{(2)}+x^{2}\left(M_{0}^{\prime 2}-M_{0}^{\prime \prime 2}\right)\right. \\
& \left.+x(1-x) q \cdot P+\left(2 A_{2}^{(1)}-1\right)\left(q^{2}-x M^{\prime 2}-x M^{\prime \prime 2}+x M_{0}^{\prime 2}+x M_{0}^{\prime \prime 2}\right)\right\} \\
& +\frac{1}{\omega \cdot P}\left(\varepsilon_{h^{\prime \prime}}^{*} \cdot P\right)\left(\varepsilon_{h^{\prime}} \cdot \omega\right) P^{\mu}\left(16 B_{1}^{(3)}+16 B_{2}^{(3)}-16 B_{1}^{(2)}+2 C_{1}^{(1)}\right) \\
& +\frac{1}{\omega \cdot P}\left(\varepsilon_{h^{\prime \prime}}^{*} \cdot \omega\right)\left(\varepsilon_{h^{\prime}} \cdot P\right) P^{\mu}\left(16 B_{1}^{(3)}-16 B_{2}^{(3)}+2 C_{1}^{(1)}\right) \\
& +\frac{1}{(\omega \cdot P)^{2}}\left(\varepsilon_{h^{\prime \prime}}^{*} \cdot \omega\right)\left(\varepsilon_{h^{\prime}} \cdot \omega\right) P^{\mu} 16 C_{3}^{(3)} \\
& +\frac{1}{\omega \cdot P}\left(\varepsilon_{h^{\prime}}^{\mu} \varepsilon_{h^{\prime \prime}}^{*} \cdot \omega+\varepsilon_{h^{\prime \prime}}^{* \mu} \varepsilon_{h^{\prime}} \cdot \omega\right)\left\{-8 C_{3}^{(3)}-4 Y+4 m^{2} C_{1}^{(1)}\right\} \\
& +\frac{1}{\omega \cdot P}\left(\varepsilon_{h^{\prime}}^{\mu} \varepsilon_{h^{\prime \prime}}^{*} \cdot \omega-\varepsilon_{h^{\prime \prime}}^{* \mu} \varepsilon_{h^{\prime}} \cdot \omega\right)\left\{-2\left(N_{1}^{\prime}-N_{1}^{\prime \prime}\right) C_{1}^{(1)}\right\} \\
& +q^{\mu}\{\cdots\}+\omega^{\mu}\{\cdots\} \text {, } \\
& B^{\mu}=\varepsilon_{h^{\prime \prime}}^{*} \cdot \varepsilon_{h^{\prime}} P^{\mu} 16 m x A_{1}^{(2)} \\
& +\left(\varepsilon_{h^{\prime \prime}}^{*} \cdot P\right)\left(\varepsilon_{h^{\prime}} \cdot P\right) P^{\mu} 2 m\left\{8 x\left(A_{2}^{(2)}-A_{4}^{(2)}+A_{2}^{(1)}-A_{1}^{(1)}\right)-2\left(A_{2}^{(1)}-A_{1}^{(1)}\right)\right\} \\
& +\left(\varepsilon_{h^{\prime}}^{\mu} \varepsilon_{h^{\prime \prime}}^{*} \cdot P+\varepsilon_{h^{\prime \prime}}^{* \mu} \varepsilon_{h^{\prime}} \cdot P\right) 2 m\left\{16 A_{1}^{(3)}-8 A_{1}^{(2)}\right. \\
& \left.-\left(A_{2}^{(1)}-A_{1}^{(1)}\right)\left(q^{2}-2 x M^{\prime \prime 2}+2 x M_{0}^{\prime \prime 2}-q \cdot P\right)\right\} \\
& +\left(\varepsilon_{h^{\prime}}^{\mu} \varepsilon_{h^{\prime \prime}}^{*} \cdot P-\varepsilon_{h^{\prime \prime}}^{* \mu} \varepsilon_{h^{\prime}} \cdot P\right) 2 m\left\{16 A_{2}^{(3)}-8 A_{1}^{(2)}\right. \\
& \left.+\left(A_{2}^{(1)}-A_{1}^{(1)}\right)\left(q^{2}-2 x M^{\prime \prime 2}+2 x M_{0}^{\prime \prime 2}-q \cdot P\right)\right\} \\
& +\frac{1}{\omega \cdot P}\left(\varepsilon_{h^{\prime \prime}}^{*} \cdot P\right)\left(\varepsilon_{h^{\prime}} \cdot \omega\right) P^{\mu} 2 m\left\{16 B_{1}^{(3)}+16 B_{2}^{(3)}-16 B_{1}^{(2)}+2 C_{1}^{(1)}\right\} \\
& +\frac{1}{\omega \cdot P}\left(\varepsilon_{h^{\prime \prime}}^{*} \cdot \omega\right)\left(\varepsilon_{h^{\prime}} \cdot P\right) P^{\mu} 2 m\left\{16 B_{1}^{(3)}-16 B_{2}^{(3)}\right\}
\end{aligned}
$$




$$
\begin{aligned}
& +\frac{1}{(\omega \cdot P)^{2}}\left(\varepsilon_{h^{\prime \prime}}^{*} \cdot \omega\right)\left(\varepsilon_{h^{\prime}} \cdot \omega\right) P^{\mu} 32 m C_{3}^{(3)} \\
& +\frac{1}{\omega \cdot P}\left(\varepsilon_{h^{\prime}}^{\mu} \varepsilon_{h^{\prime \prime}}^{*} \cdot \omega+\varepsilon_{h^{\prime \prime}}^{* \mu} \varepsilon_{h^{\prime}} \cdot \omega\right) 2 m\left\{-8 C_{3}^{(3)}-4 Y+4 m^{2} C_{1}^{(1)}\right. \\
& \left.+2 N_{1}^{\prime} C_{1}^{(1)}-\left(q^{2}+q \cdot P\right) C_{1}^{(1)}\right\} \\
& +\frac{1}{\omega \cdot P}\left(\varepsilon_{h^{\prime}}^{\mu} \varepsilon_{h^{\prime \prime}}^{*} \cdot \omega-\varepsilon_{h^{\prime \prime}}^{* \mu} \varepsilon_{h^{\prime}} \cdot \omega\right) 2 m\left\{2 N_{1}^{\prime \prime} C_{1}^{(1)}-\left(q^{2}-q \cdot P\right) C_{1}^{(1)}\right\} \\
& +q^{\mu}\{\cdots\}+\omega^{\mu}\{\cdots\} \\
& C^{\mu}=\varepsilon_{h^{\prime \prime}}^{*} \cdot \varepsilon_{h^{\prime}} P^{\mu} 16 m x A_{1}^{(2)} \\
& +\left(\varepsilon_{h^{\prime \prime}}^{*} \cdot P\right)\left(\varepsilon_{h^{\prime}} \cdot P\right) P^{\mu} 2 m\left\{8 x\left(A_{2}^{(2)}-A_{4}^{(2)}+A_{2}^{(1)}-A_{1}^{(1)}\right)\right. \\
& \left.+2\left(A_{2}^{(1)}+A_{1}^{(1)}-1\right)\right\} \\
& +\left(\varepsilon_{h^{\prime}}^{\mu} \varepsilon_{h^{\prime \prime}}^{*} \cdot P+\varepsilon_{h^{\prime \prime}}^{* \mu} \varepsilon_{h^{\prime}} \cdot P\right) 2 m\left\{16 A_{1}^{(3)}-8 A_{1}^{(2)}\right. \\
& \left.+\left(A_{2}^{(1)}+A_{1}^{(1)}-1\right)\left(q^{2}-2 x M^{\prime 2}+2 x M_{0}^{\prime 2}+q \cdot P\right)\right\} \\
& +\left(\varepsilon_{h^{\prime}}^{\mu} \varepsilon_{h^{\prime \prime}}^{*} \cdot P-\varepsilon_{h^{\prime \prime}}^{* \mu} \varepsilon_{h^{\prime}} \cdot P\right) 2 m\left\{16 A_{2}^{(3)}-8 A_{1}^{(2)}\right. \\
& \left.+\left(A_{2}^{(1)}+A_{1}^{(1)}-1\right)\left(q^{2}-2 x M^{\prime 2}+2 x M_{0}^{\prime 2}+q \cdot P\right)\right\} \\
& +\frac{1}{\omega \cdot P}\left(\varepsilon_{h^{\prime \prime}}^{*} \cdot P\right)\left(\varepsilon_{h^{\prime}} \cdot \omega\right) P^{\mu} 2 m\left\{16 B_{1}^{(3)}+16 B_{2}^{(3)}-16 B_{1}^{(2)}\right\} \\
& +\frac{1}{\omega \cdot P}\left(\varepsilon_{h^{\prime \prime}}^{*} \cdot \omega\right)\left(\varepsilon_{h^{\prime}} \cdot P\right) P^{\mu} 2 m\left\{16 B_{1}^{(3)}-16 B_{2}^{(3)}+2 C_{1}^{(1)}\right\} \\
& +\frac{1}{(\omega \cdot P)^{2}}\left(\varepsilon_{h^{\prime \prime}}^{*} \cdot \omega\right)\left(\varepsilon_{h^{\prime}} \cdot \omega\right) P^{\mu} 32 m C_{3}^{(3)} \\
& +\frac{1}{\omega \cdot P}\left(\varepsilon_{h^{\prime}}^{\mu} \varepsilon_{h^{\prime \prime}}^{*} \cdot \omega+\varepsilon_{h^{\prime \prime}}^{* \mu} \varepsilon_{h^{\prime}} \cdot \omega\right) 2 m\left\{-8 C_{3}^{(3)}-4 Y+4 m^{2} C_{1}^{(1)}\right. \\
& \left.+2 N_{1}^{\prime \prime} C_{1}^{(1)}-\left(q^{2}-q \cdot P\right) C_{1}^{(1)}\right\} \\
& +\frac{1}{\omega \cdot P}\left(\varepsilon_{h^{\prime}}^{\mu} \varepsilon_{h^{\prime \prime}}^{*} \cdot \omega-\varepsilon_{h^{\prime \prime}}^{* \mu} \varepsilon_{h^{\prime}} \cdot \omega\right) 2 m\left\{-2 N_{1}^{\prime} C_{1}^{(1)}+\left(q^{2}+q \cdot P\right) C_{1}^{(1)}\right\} \\
& +q^{\mu}\{\cdots\}+\omega^{\mu}\{\cdots\} \\
& D^{\mu}=\varepsilon_{h^{\prime \prime}}^{*} \cdot \varepsilon_{h^{\prime}} P^{\mu} 4 A_{1}^{(2)}\left\{x\left(8 m^{2}-M_{0}^{\prime 2}-M_{0}^{\prime \prime 2}\right)-(1-x) q^{2}\right\} \\
& +\left(\varepsilon_{h^{\prime \prime}}^{*} \cdot P\right)\left(\varepsilon_{h^{\prime}} \cdot P\right) P^{\mu} 4\left(A_{2}^{(2)}-A_{2}^{(4)}+A_{2}^{(1)}-A_{1}^{(1)}\right)\left\{x\left(8 m^{2}-M_{0}^{\prime 2}-M_{0}^{\prime \prime 2}\right)\right. \\
& \left.-(1-x) q^{2}\right\}
\end{aligned}
$$




$$
\begin{aligned}
& +\left(\varepsilon_{h^{\prime}}^{\mu} \varepsilon_{h^{\prime \prime}}^{*} \cdot P+\varepsilon_{h^{\prime \prime}}^{* \mu} \varepsilon_{h^{\prime}} \cdot P\right)\left\{4(1-x) A_{1}^{(2)}\left(M^{\prime 2}+M^{\prime \prime 2}-q^{2}-8 m^{2}\right)\right. \\
& \left.-8 A_{1}^{(2)} Z_{2}+8 C_{1}^{(3)}\right\} \\
& +\left(\varepsilon_{h^{\prime}}^{\mu} \varepsilon_{h^{\prime \prime}}^{*} \cdot P-\varepsilon_{h^{\prime \prime}}^{* \mu} \varepsilon_{h^{\prime}} \cdot P\right)\left\{4 ( 2 A _ { 2 } ^ { ( 3 ) } - A _ { 1 } ^ { ( 2 ) } ) \left(q^{2}-M^{\prime 2}-M^{\prime \prime 2}\right.\right. \\
& \left.\left.+2 N_{2}+8 m^{2}\right)\right\} \\
& +\frac{1}{\omega \cdot P}\left(\varepsilon_{h^{\prime \prime}}^{*} \cdot P\right)\left(\varepsilon_{h^{\prime}} \cdot \omega\right) P^{\mu}\left\{8 B_{1}^{(3)}\left(q^{2}-M^{\prime 2}-M^{\prime \prime 2}+8 m^{2}\right)\right. \\
& +8\left(B_{2}^{(3)}-B_{1}^{(2)}\right)\left(q^{2}-M^{\prime 2}-M^{\prime \prime 2}+8 m^{2}+2 N_{2}\right) \\
& \left.-4\left(B_{1}^{(2)}+C_{1}^{(2)}-C_{1}^{(1)}\right)\left(q^{2}-N_{1}^{\prime}-N_{1}^{\prime \prime}\right)\right\} \\
& +\frac{1}{\omega \cdot P}\left(\varepsilon_{h^{\prime \prime}}^{*} \cdot \omega\right)\left(\varepsilon_{h^{\prime}} \cdot P\right) P^{\mu}\left\{8 B_{1}^{(3)}\left(q^{2}-M^{\prime 2}-M^{\prime \prime 2}+8 m^{2}\right)\right. \\
& \left.-8 B_{2}^{(3)}\left(q^{2}-M^{\prime 2}-M^{\prime \prime 2}+8 m^{2}+2 N_{2}\right)-4\left(B_{1}^{(2)}-C_{1}^{(2)}\right)\left(q^{2}-N_{1}^{\prime}-N_{1}^{\prime \prime}\right)\right\} \\
& +\frac{1}{(\omega \cdot P)^{2}}\left(\varepsilon_{h^{\prime \prime}}^{*} \cdot \omega\right)\left(\varepsilon_{h^{\prime}} \cdot \omega\right) P^{\mu}\left\{8 C_{3}^{(3)}\left(q^{2}-M^{\prime 2}-M^{\prime \prime 2}+8 m^{2}+2 N_{2}\right)\right. \\
& \left.-4 C_{2}^{(2)}\left(q^{2}-N_{1}^{\prime}-N_{1}^{\prime \prime}\right)\right\} \\
& +\frac{1}{\omega \cdot P}\left(\varepsilon_{h^{\prime}}^{\mu} \varepsilon_{h^{\prime \prime}}^{*} \cdot \omega+\varepsilon_{h^{\prime \prime}}^{* \mu} \varepsilon_{h^{\prime}} \cdot \omega\right) 8 C_{1}^{(3)}\left\{q^{2}-M^{\prime 2}-M^{\prime \prime 2}+8 m^{2}+2 N_{2}\right\} \\
& +q^{\mu}\{\cdots\}+\omega^{\mu}\{\cdots\}, \\
& \cdots
\end{aligned}
$$

where we have used the relation (A.7) and $Y$ is given by Eq.(2.26). The trace $D^{\mu}$ contains higher order momentum contributions which we have not analyzed in detail, but those contributions that are required for the computation of the form factors $F_{1}\left(q^{2}\right), F_{2}\left(q^{2}\right)$ and $F_{3}\left(q^{2}\right)$ are unambiguously determined by our approach.

\section{Appendix: Determination of $f\left(q^{2}\right)$ and $f_{V}$ in the SLF and CDKM schemes}

First we shall derive the formulas for $f\left(q^{2}\right)$. Using the tensor decomposition of Appendix A the traces of Eq.(3.6) can be represented as

$$
\begin{aligned}
\hat{A}^{\mu}= & \varepsilon_{h^{\prime \prime}}^{* \mu}\left\{-2 m_{1}^{\prime \prime}\left(M^{\prime 2}-N_{1}^{\prime}-N_{2}-\left(m_{1}^{\prime}-m_{2}\right)^{2}\right)-8\left(m_{1}^{\prime}-m_{2}\right) A_{1}^{(2)}\right. \\
& -2 m_{1}^{\prime}\left(M^{\prime \prime 2}-N_{1}^{\prime \prime}-N_{2}-m_{1}^{\prime \prime 2}-m_{2}^{2}\right) \\
& \left.+2 m_{2}\left(q^{2}-N_{1}^{\prime}-N_{1}^{\prime \prime}-m_{1}^{\prime 2}-m_{1}^{\prime \prime 2}\right)\right\} \\
& +P^{\mu} \varepsilon_{h^{\prime \prime}}^{*} \cdot P\left\{-8\left(m_{1}^{\prime}-m_{2}\right)\left(A_{2}^{(2)}+A_{3}^{(2)}\right)+2\left(m_{1}^{\prime}+m_{1}^{\prime \prime}\right) A_{2}^{(1)}\right. \\
& \left.+2\left(5 m_{1}^{\prime}-m_{1}^{\prime \prime}-2 m_{2}\right) A_{1}^{(1)}-2 m_{1}^{\prime}\right\}
\end{aligned}
$$




$$
\begin{aligned}
& +P^{\mu} \frac{\varepsilon_{h^{\prime \prime}}^{*} \cdot \omega}{\omega \cdot P}\left\{8\left(m_{2}-m_{1}^{\prime}\right) B_{1}^{(2)}+2\left(m_{1}^{\prime}+m_{1}^{\prime \prime}\right) C_{1}^{(1)}\right\} \\
& +q^{\mu}\{\cdots\}+\omega^{\mu}\{\cdots\}, \\
\hat{B}^{\mu}= & \varepsilon_{h^{\prime \prime}}^{* \mu} 4 A_{1}^{(2)}\left\{M^{\prime 2}+M^{\prime \prime 2}-q^{2}-2 N_{2}+2\left(m_{1}^{\prime}-m_{2}\right)\left(m_{1}^{\prime \prime}+m_{2}\right)\right\} \\
& +P^{\mu} \varepsilon_{h^{\prime \prime}}^{*} \cdot P\left\{4 \left(M^{2}+M^{\prime \prime 2}-q^{2}\right.\right. \\
& \left.+2\left(m_{1}^{\prime}-m_{2}\right)\left(m_{1}^{\prime \prime}+m_{2}\right)\right)\left(A_{2}^{(2)}+A_{3}^{(2)}-A_{1}^{(1)}\right) \\
& \left.+2\left(q^{2}-N_{1}^{\prime}-N_{1}^{\prime \prime}-\left(m_{1}^{\prime}+m_{1}^{\prime \prime}\right)^{2}\right)\left(A_{1}^{(1)}+A_{2}^{(1)}-1\right)\right\} \\
& +P^{\mu} \frac{\varepsilon_{h^{\prime \prime}}^{*} \cdot \omega}{\omega \cdot P}\left\{4\left(M^{\prime 2}+M^{\prime \prime 2}-q^{2}-2 N_{2}+2\left(m_{1}^{\prime}-m_{2}\right)\left(m_{1}^{\prime \prime}+m_{2}\right)\right) B_{1}^{(2)}\right. \\
& \left.+2\left(q^{2}-N_{1}^{\prime}-N_{1}^{\prime \prime}-\left(m_{1}^{\prime}+m_{1}^{\prime \prime}\right)^{2}\right) C_{1}^{(1)}\right\} \\
& +q^{\mu}\{\cdots\}+\omega^{\mu}\{\cdots\},
\end{aligned}
$$

where we have used the relation (A.7).

In the standard light-front formalism only $\hat{A}^{+}$and $\hat{B}^{+}$are used with the additional requirement that all quarks are on their respective mass shells, i.e., $N_{1}^{\prime}, N_{1}^{\prime \prime}$ and $N_{2}$ are put equal to zero and $M^{\prime}, M^{\prime \prime}$ are replaced by $M_{0}^{\prime}, M_{0}^{\prime \prime}$, respectively, on the right-hand-side of Eq.(C.1), but $P^{\prime+}=P^{\prime \prime+}$ is kept unchanged. In particular, $\hat{G}_{0}^{+}=-2 P^{\prime+} A_{0}\left(q^{2}\right)$ is given by

$$
\begin{aligned}
A_{0}\left(q^{2}\right)= & \frac{N_{c}}{16 \pi^{3}} \int_{0}^{1} d x \int d^{2} p_{\perp}^{\prime} \frac{2 h_{0}^{\prime} h_{0}^{\prime \prime}}{(1-x) \hat{N}_{1}^{\prime} \hat{N}_{1}^{\prime \prime}}\left\{2 x M_{0}^{\prime \prime}\left[(1-x) m_{1}^{\prime}+x m_{2}\right)\right] \\
& +\frac{(2 x-1) M_{0}^{\prime \prime}+m_{1}^{\prime \prime}-m_{2}}{(1-x) D^{\prime \prime}}\left[p_{\perp}^{\prime} p_{\perp}^{\prime \prime}\right. \\
& \left.\left.+\left(x m_{2}+(1-x) m_{1}^{\prime}\right)\left(x m_{2}-(1-x) m_{1}^{\prime \prime}\right)\right]\right\},
\end{aligned}
$$

and the SLF expression for the form factor $f\left(q^{2}\right)$, denoted as $f^{S L F}\left(q^{2}\right)$, is

$$
f^{S L F}\left(q^{2}\right)=-2 M^{\prime \prime} A_{0}\left(q^{2}\right)-\left(M^{2}-M^{\prime \prime 2}-q^{2}\right) a_{+}\left(q^{2}\right) .
$$

Yet another expression for $f\left(q^{2}\right)$ is found if the amplitude $\hat{G}_{+}^{\mu}$ for on-shell quarks is used, i.e., if the longitutinal mode is avoided. We denote it by $f^{C D K M}\left(q^{2}\right)$ since the CDKM approach of Ref. [2], gives the same result:

$$
\begin{aligned}
f^{C D K M}\left(q^{2}\right)= & \frac{N_{c}}{16 \pi^{3}} \int_{0}^{1} d x \int d^{2} p_{\perp}^{\prime} \frac{h_{0}^{\prime} h_{0}^{\prime \prime}}{(1-x) \hat{N}_{1}^{\prime} \hat{N}_{1}^{\prime \prime}}\left\{-2 m_{1}^{\prime \prime} M_{0}^{\prime 2}-2 m_{1}^{\prime} M_{0}^{\prime \prime 2}+2 m_{2} q^{2}\right. \\
& +2\left(m_{1}^{\prime}-m_{2}\right)\left(m_{1}^{\prime \prime}-m_{2}\right)\left(m_{1}^{\prime}+m_{1}^{\prime \prime}\right)-8\left(m_{1}^{\prime}-m_{2}\right) A_{1}^{(2)} \\
& \left.+\frac{4 A_{1}^{(2)}}{D^{\prime \prime}}\left[M_{0}^{\prime 2}+M_{0}^{\prime \prime 2}-q^{2}+2\left(m_{1}^{\prime}-m_{2}\right)\left(m_{1}^{\prime \prime}+m_{2}\right)\right]\right\} .
\end{aligned}
$$

Next we shall derive analoguous formulas for $f_{V}$. Using the tensor decompositions of Appendix A the trace (3.13) can be represented as

$$
s^{\mu}=\varepsilon_{h}^{\mu}\left\{8 A_{1}^{(2)}+2\left(M^{2}-N_{1}^{\prime}-N_{2}-\left(m_{1}-m_{2}\right)^{2}\right)\right\}
$$




$$
\begin{aligned}
& +P^{\mu} \frac{\varepsilon_{h} \cdot \omega}{\omega \cdot P}\left\{8 B_{1}^{(2)}-2 C_{1}^{(1)}\right\}+\omega^{\mu} \frac{\varepsilon_{h} \cdot \omega}{(\omega \cdot P)^{2}} 2 C_{2}^{(2)} \\
& -\frac{2}{D^{\prime}}\left\{\varepsilon_{h}^{\mu} 4\left(m_{1}+m_{2}\right) A_{1}^{(2)}\right. \\
& \left.+P^{\mu} \frac{\varepsilon_{h} \cdot \omega}{\omega \cdot P}\left(4\left(m_{1}+m_{2}\right) B_{1}^{(2)}-2 m_{1} C_{1}^{(1)}\right)+\omega^{\mu} \frac{\varepsilon_{h} \cdot \omega}{(\omega \cdot P)^{2}}\left(m_{1}+m_{2}\right) C_{2}^{(2)}\right\} .
\end{aligned}
$$

The standard light-front expression for the vector decay constant, $f_{V}^{S L F}$, can be derived for on-shell quarks [20]:

$$
\begin{array}{r}
f_{V}^{S L F}=\frac{N_{c}}{8 \pi^{3}} \int_{0}^{1} d x \int d^{2} p_{\perp}^{\prime} \frac{\sqrt{2} h_{0}^{\prime}}{(1-x) \hat{N}_{1}^{\prime}} \frac{M}{M_{0}^{\prime}}\left\{x M_{0}^{\prime 2}-m_{1}^{\prime}\left(m_{1}^{\prime}-m_{2}\right)-p_{\perp}^{\prime 2}\right. \\
\left.+\frac{m_{1}^{\prime}+m_{2}}{D^{\prime}} p_{\perp}^{\prime 2}\right\} .
\end{array}
$$

A derivation based upon the amplitude $\hat{g}_{+}^{\perp}$, again for on-shell quarks, or that uses the approach of Ref. [2] leads to

$$
\begin{array}{r}
f_{V}^{C D K M}=\frac{N_{c}}{8 \pi^{3}} \int_{0}^{1} d x \int d^{2} p_{\perp}^{\prime} \frac{\sqrt{2} h_{0}^{\prime}}{(1-x) \hat{N}_{1}^{\prime}}\left\{x M_{0}^{\prime 2}-m_{1}^{\prime}\left(m_{1}^{\prime}-m_{2}\right)-p_{\perp}^{\prime 2}\right. \\
\left.+\frac{m_{1}^{\prime}+m_{2}}{D^{\prime}} p_{\perp}^{\prime 2}\right\} .
\end{array}
$$

\section{References}

[1] M.V.Terent'ev, Yad. Fiz. 24, 207 (1976) [Sov. J. Nucl. Phys. 24, 106 (1976)]; V.J.Berestetsky, and M.V.Terent'ev, ibid. 24, 1044 (1976) [24, 547 (1976)]; ibid. 25, 653 (1977) [25, 347 (1977)].

[2] V. A. Karmanov, and A. V. Smirnov, Nucl. Phys. A546, 691 (1992);

J. Carbonell, B. Desplanques, V. A. Karmanov, and J. F. Mathiot, Phys. Rep. 300, 215 (1998).

[3] M. G. Fuda, Ann. Phys. 197, 265 (1990).

[4] A. Szczepaniak, C R. Ji, and S. R. Cotanch, Phys. Rev. D52, 5284 (1995).

[5] H. Leutwyler, and J. Stern, Ann. Phys. 112, 94 (1978).

[6] L. L. Frankfurt, and M. I. Strikman, Nucl. Phys. B148, 107 (1979); Phys. Rep. 76, 215 (1981);

L. L. Frankfurt, I. L. Grach, L. A. Kondratyuk, and M. I. Strikman, Phys. Rev. Lett. 62, 387 (1989);

L. L. Frankfurt, T. Frederico, and M. Strikman, Phys. Rev. C48, 2182 (1993);

B. D. Keister, Phys. Rev. D49, 1500 (1994). 
[7] S. J. Chang, R. G. Root, and T. M. Yan, Phys. Rev. D7, 1133 (1973); T. M. Yan, Phys. Rev. D7, 1780 (1973).

[8] W. Jaus, Phys. Rev. D60, 054026 (1999).

[9] J. P. B. C. de Melo, J. H. O. Sales, T. Frederico, and P. U. Sauer, Nucl. Phys. A631, 574c (1998);

J. P. B. C. de Melo, T. Frederico, H. W. L. Naus, and P. U. Sauer, Nucl. Phys. A660, 219 (1999).

[10] S. J. Brodsky, and D. S. Hwang, hep-ph/9806358.

[11] H. M. Choi, and C. R. Ji, Phys. Rev. D58, 071901 (1998).

[12] I. L. Grach, and L. A. Kondratyuk, Sov. J. Nucl. Phys. 39,198 (1984).

[13] P. L. Chung, F. Coester, B. D. Keister, and W. N. Polyzou, Phys. Rev. C37, 2000 (1988).

[14] S. J. Brodsky, and J. R. Hiller, Phys. Rev. D46, 2141 (1992).

[15] B. L. G. Bakker, and C.-R. Ji, Phys. Rev. D65, (2002)[hep-ph/0109005].

[16] H.B. L. G. Bakker,H.- M. Choi, and C.-R. Ji, hep-ph/0202217.

[17] W. Jaus, Phys. Rev. D53, 1349 (1996); ibid. D54, 5904 (E) (1996).

[18] D. Melikhov, and S. Simula, hep-ph/0112044.

[19] W. Jaus, Phys. Rev. D41, 3394 (1990).

[20] W. Jaus, Phys. Rev. D44, 2851 (1991).

[21] H.-M. Choi, and C.-R. Ji, Nucl. Phys. A618, 291 (1997).

[22] H.-M. Choi, and C.-R. Ji, Phys. Rev. D59, 074015 (1999).

[23] K. Hagiwara et al., Phys. Rev. D66, 01001 (2002).

[24] S. Ryan, Nucl. Phys. B106 (Proc. Suppl.), 86 (2002).

[25] S. Narison, hep-ph/0202200.

[26] A.A. Penin, and M. Steinhauser, Phys. Rev. D65, 054006 (2002).

[27] D. Becirevic et al., hep-lat/9811003.

[28] C. Bernard et al., hep-lat/9806412.

[29] F. Cardarelli et al., Phys. Lett. B349, 393 (1995). 
[30] R. Shtokhamer, and P. Singer, Phys. Rev. D7, 790 (1973).

[31] N. Kroll,T. Lee, and B. Zumino, Phys. Rev. 157, 1376 (1967).

[32] A. Samsonov, hep-ph/0208165.

[33] F. Hawes, and M. Pichowsky, Phys. Rev. C59, 1743 (1999).

[34] M. Hecht, and B.H.J. McKellar, Phys. Rev. C57, 2638 (1998).

[35] N. Levy, M. Glück, and S. Wagner, Phys. Rev. D4, 874 (1971).

[36] J.J.J. Kokkedee, The quark model (Benjamin, 1969).

[37] N. Isgur, Phys. Rev. Lett. 36, 1262 (1976).

[38] T. Das, V. S. Mathur, and S. Okubo, Phys. Rev. Lett. 19, 1067 (1967).

[39] P. J. O’Donnell, and Q. P. Xu, Phys. Lett. B336, 113 (1994).

[40] T. M. Yan et al., Phys. Rev. D46, 1148 (1992); D55, 5851(E) (1997).

[41] B. Desplanques, V. A. Karmanov, and J. F. Mathiot, Nucl. Phys. A589, 697 (1995).

[42] V. A. Karmanov, and J. F. Mathiot, Nucl. Phys. A602, 388 (1996).,

[43] CLEO Collaboration, S. Ahmed et al., Phys. Rev. Lett. 87, 251801 (2001).

[44] D. Becirevic, and A. Le Yaouanc, JHEP 9903, 021 (1999).

[45] P. Singer, hep-ph/9910558, D. Guetta, and P. Singer, hep-ph/0009057.

[46] A. Abada et al., hep-ph/0206237.

[47] P. Colangelo, and A. Khodjamirian, hep-ph/0010175.

[48] A. Khodjamirian, R. Ruckl, S. Weinzierl, and O. I. Yakovlev, Phys. Lett. B457, 245 (1999).

[49] Hungchong Kim, hep-ph/0206170.

[50] D. Becirevic et al., hep-ph/0212177.

[51] H. Leutwyler, Cargese 1996, Masses of fundamental particles, 149, and hep$\mathrm{ph} / 9609467$.

[52] L. S. Kisslinger, H.-M. Choi, and C.-R. Ji, Phys.Rev. D63, 113005 (2001).

[53] H. Leutwyler, Phys. Lett. B378, 313 (1996). 